\title{
CASIMIR OPERATORS AND MONODROMY REPRESENTATIONS OF GENERALISED BRAID GROUPS
}

\author{
JOHN J. MILLSON* AND VALERIO TOLEDANO LAREDO**
}

\begin{abstract}
Let $\mathfrak{g}$ be a complex, simple Lie algebra with Cartan subalgebra $\mathfrak{h}$ and Weyl group $W$. We construct a one-parameter family of flat connections $\nabla_{\kappa}$ on $\mathfrak{h}$ with values in any finite-dimensional $\mathfrak{g}$-module $V$ and simple poles on the root hyperplanes. The corresponding monodromy representation of the braid group $B_{\mathfrak{g}}$ of type $\mathfrak{g}$ is a deformation of the action of (a finite extension of) $W$ on $V$. The residues of $\nabla_{\kappa}$ are the Casimirs $\kappa_{\alpha}$ of the subalgebras $\mathfrak{s l}_{2}^{\alpha} \subset \mathfrak{g}$ corresponding to the roots of $\mathfrak{g}$. The irreducibility of a subspace $U \subseteq V$ under the $\kappa_{\alpha}$ implies that, for generic values of the parameter, the braid group $B_{\mathfrak{g}}$ acts irreducibly on $U$. Answering a question of Knutson and Procesi, we show that these Casimirs act irreducibly on the weight spaces of all simple $\mathfrak{g}$-modules if $\mathfrak{g}=\mathfrak{s l}_{3}$ but that this is not the case if $\mathfrak{g} ¥ \mathfrak{s l}_{2}, \mathfrak{s l}_{3}$. We use this to disprove a conjecture of Kwon and Lusztig stating the irreducibility of quantum Weyl group actions of Artin's braid group $B_{n}$ on the zero weight spaces of all simple $U_{\hbar} \mathfrak{s l}_{n}-$ modules for $n \geq 4$. Finally, we study the irreducibility of the action of the Casimirs on the zero weight spaces of self-dual $\mathfrak{g}$-modules and obtain complete classification results for $\mathfrak{g}=\mathfrak{s l}_{n}$ and $\mathfrak{g}_{2}$.
\end{abstract}

\section{Contents}

1. Introduction

2. Flat connections on $\mathfrak{h}_{\text {reg }} \quad 6$

3. Generic irreducibility of monodromy representations 14

4. The Casimir algebra $\mathcal{C}_{\mathfrak{g}}$ of $\mathfrak{g} \quad 20$

5. A conjecture of Kwon and Lusztig on quantum Weyl groups 25

6. Irreducible representations of $\mathcal{C}_{\mathfrak{g}} \quad 28$

7. Zero weight spaces of self-dual $\mathfrak{g}$-modules 30

8. Appendix : The centraliser of the Casimir algebra 38

References

Date: September 2004.

* work partially supported by NSF grants DMS-98-03520 and 01-04006.

** work partially supported by an MSRI postdoctoral fellowship for the year 2000-2001. 


\section{INTRODUCTION}

It has been known since the seminal work of Knizhnik and Zamolodchikov how to construct representations of Artin's braid groups $B_{n}$ by using the representation theory of a given complex, semi-simple Lie algebra $\mathfrak{g}[\mathrm{KZ}]$. Realising $B_{n}$ as the fundamental group of the quotient of the configuration space

$$
X_{n}=\left\{\left(z_{1}, \ldots, z_{n}\right) \in \mathbb{C}^{n} \mid z_{i} \neq z_{j}, 1 \leq i<j \leq n\right\}
$$

by the natural action of the symmetric group $\mathfrak{S}_{n}$, one obtains these representations as the monodromy of the Knizhnik-Zamolodchikov connection

$$
\nabla_{\mathrm{KZ}}=d-h \sum_{1 \leq i<j \leq n} \frac{d\left(z_{i}-z_{j}\right)}{z_{i}-z_{j}} \cdot \Omega_{i j}
$$

with values in the $n$-fold tensor product $V^{\otimes n}$ of a finite-dimensional $\mathfrak{g}-$ module $V$. Here, the one-form $\nabla_{\mathrm{Kz}}$ is regarded as an $\mathfrak{S}_{n}$-equivariant flat connection on the topologically trivial vector bundle over $X_{n}$ with fibre $V^{\otimes n}$ and then pushed down to $X_{n} / \mathfrak{S}_{n}$. Its coefficients $\Omega_{i j} \in \operatorname{End}\left(V^{\otimes n}\right)$ are given by

$$
\Omega_{i j}=\sum_{a=1}^{\operatorname{dim} \mathfrak{g}} \pi_{i}\left(X_{a}\right) \pi_{j}\left(X^{a}\right)
$$

where $\left\{X_{a}\right\},\left\{X^{a}\right\}$ are dual basis of $\mathfrak{g}$ with respect to the Killing form and $\pi_{k}(\cdot)$ denotes the action on the $k$ th tensor factor of $V^{\otimes n}$. Finally, the complex number $h$ may be regarded as a deformation parameter which, upon being set to 0 , gives a monodromy representation of $B_{n}$ factoring through the natural action of the symmetric group on $V^{\otimes n}$.

Aside from their intrinsic interest, these representations appear naturally in a number of different contexts. They define for example the commutativity and associativity constraints in the tensor category of highest weight representations of the affine Kac-Moody algebra $\widehat{\mathfrak{g}}$ [KL, Wa, TL1] and, by the Kohno-Drinfeld theorem, on the finite-dimensional representations of the quantum group $U_{\hbar} \mathfrak{g}[\mathrm{Dr} 3, \mathrm{Dr} 4, \mathrm{Ko} 1]$. As such, they define invariants of knots and links and, for suitable rational values of $h$, of three-manifolds [Tu].

The purpose of the present paper is to use the representation theory of $\mathfrak{g}$ in a similar vein to construct monodromy representations of a different braid group, namely the generalised braid group $B_{\mathfrak{g}}$ of type $\mathfrak{g}$. The latter may be defined as the fundamental group of the quotient $\mathfrak{h}_{\text {reg }} / W$ of the set $\mathfrak{h}_{\text {reg }}$ of regular elements in a Cartan subalgebra $\mathfrak{h}$ of $\mathfrak{g}$ by the action of the corresponding Weyl group $W$. Like Artin's braid groups, $B_{\mathfrak{g}}$ is presented on generators $S_{1}, \ldots, S_{n}$ labelled by a choice $\alpha_{1}, \ldots, \alpha_{n}$ of simple roots of $\mathfrak{g}$ with relations

$$
S_{i} S_{j} \cdots=S_{j} S_{i} \cdots
$$


for any $i \neq j$, where the number of factors on each side is equal to the order of the product $s_{i} s_{j}$ of the orthogonal reflections corresponding to $\alpha_{i}$ and $\alpha_{j}$ in $W[\mathrm{Br}]$.

To state our first main result, let $R=\{\alpha\} \subset \mathfrak{h}^{*}$ be the set of roots of $\mathfrak{g}$ relative to $\mathfrak{h}$ so that $\mathfrak{h}_{\text {reg }}=\mathfrak{h} \backslash \bigcup_{\alpha \in R} \operatorname{Ker}(\alpha)$. For each $\alpha \in R$, let $\mathfrak{s l}_{2}^{\alpha}=$ $\left\langle e_{\alpha}, f_{\alpha}, h_{\alpha}\right\rangle \subseteq \mathfrak{g}$ be the corresponding $\mathfrak{s l}_{2}(\mathbb{C})$-subalgebra of $\mathfrak{g}$ and

$$
\kappa_{\alpha}=\frac{\langle\alpha, \alpha\rangle}{2}\left(e_{\alpha} f_{\alpha}+f_{\alpha} e_{\alpha}\right)
$$

the truncated Casimir operator of $\mathfrak{s l}_{2}^{\alpha}$ where $\langle\cdot, \cdot\rangle$ is a fixed multiple of the Killing form of $\mathfrak{g}$. Let $V$ be a $\mathfrak{g}$-module, then we prove in section 2 the following ${ }^{1}$

Theorem 1.1. The one-form

$$
\nabla_{\kappa}=d-h \sum_{\alpha \in R} \frac{d \alpha}{\alpha} \cdot \kappa_{\alpha}
$$

defines, for any $h \in \mathbb{C}$, a flat connection on the trivial vector bundle over $\mathfrak{h}_{\mathrm{reg}}$ with fibre $V$, which is reducible with respect to the weight space decomposition of $V$.

As a consequence, each weight space of $V$ carries a canonical one-parameter family of monodromy representations of the pure braid group $P_{\mathfrak{g}}=\pi_{1}\left(\mathfrak{h}_{\text {reg }}\right)$. This action extends to one of the full braid group $B_{\mathfrak{g}}$ on the direct sum of weight spaces corresponding to a given Weyl group orbit, and in particular on the zero weight space of $V$, by pushing $\nabla_{\kappa}$ down to the quotient space $\mathfrak{h}_{\text {reg }} / W$. Since the Weyl group itself does not act on $V$, this requires choosing an action of $B_{\mathfrak{g}}$ on $V$ which permutes the weight spaces compatibly with the projection $B_{\mathfrak{g}} \rightarrow W$. This may for example be achieved by taking the simply-connected complex Lie group $G$ corresponding to $\mathfrak{g}$ and mapping $B_{\mathfrak{g}}$ to one of the Tits extension $\widetilde{W}$ of $W$, a class of subgroups of the normaliser in $G$ of the torus $T$ corresponding to $\mathfrak{h}$ which are extensions of $W$ by the sign group $\mathbb{Z}_{2}^{n}$, where $n=\operatorname{dim}(\mathfrak{h})$ [Ti]. The choice of a specific Tits extension is somewhat immaterial since any two are conjugate by an element of $T$ and the corresponding representations of $B_{\mathfrak{g}}$ are therefore equivalent.

The rest of the paper is devoted to the study of the irreducibility of our monodromy representations. Define a subspace $U \subseteq V$ invariant under the monodromy action of $B_{\mathfrak{g}}$ to be generically irreducible if it is irreducible for all values of $h$ lying outside the zero set of some holomorphic function. In section 3 , we prove the following

\footnotetext{
${ }^{1}$ Theorem 1.1 was independently discovered by De Concini around 1995 (unpublished). A variant of the connection $\nabla_{\kappa}$ also appears in the recent paper [FMTV]. Unlike $\nabla_{\kappa}$ however, the connection introduced in [FMTV] is not $W$-equivariant and therefore only defines representations of the pure braid group $P_{\mathfrak{g}}$ instead of the full braid group $B_{\mathfrak{g}}$
} 
Theorem 1.2. A subspace $U \subseteq V$ is generically irreducible under the braid group $B_{\mathfrak{g}}$ (resp. the pure braid group $P_{\mathfrak{g}}$ ) if, and only if it is irreducibly acted upon by the Casimirs $\kappa_{\alpha}$ and $\widetilde{W}$ (resp. the $\kappa_{\alpha}$ and $\left.\widetilde{W} \cap T\right)$.

This naturally prompts the question, originally asked us by C. Procesi and A. Knutson, of whether the Casimir algebra i.e., the algebra

$$
\mathcal{C}_{\mathfrak{g}}=\left\langle\kappa_{\alpha}\right\rangle_{\alpha \in R} \vee \mathfrak{h} \subset U \mathfrak{g}
$$

generated by the Casimirs $\kappa_{\alpha}$ and $\mathfrak{h}$ inside the enveloping algebra of $\mathfrak{g}$ acts irreducibly on the weight spaces of any simple $\mathfrak{g}$-module or, stronger still, whether it is equal to the algebra $U \mathfrak{g}^{\mathfrak{h}}$ of $\mathfrak{h}$-invariants in $U \mathfrak{g}$.

The answer to both questions is clearly positive for $\mathfrak{g}=\mathfrak{s l}_{2}$ and we show in section 4 that it this almost so for $\mathfrak{g}=\mathfrak{s l}_{3}$. More precisely,

Theorem 1.3. If $\mathfrak{g}=\mathfrak{s l}_{3}, \mathcal{C}_{\mathfrak{g}}$ is a proper subalgebra of $U \mathfrak{g}^{\mathfrak{h}}$, but the latter is generated by $\mathcal{C}_{\mathfrak{g}}$ and the centre $Z(U \mathfrak{g})$ of $U \mathfrak{g}$. In particular, $\mathcal{C}_{\mathfrak{g}}$ acts irreducibly on the weight spaces of any simple $\mathfrak{g}$-module.

As a consequence, all monodromy representations of $P_{3}$ on weight spaces of simple $\mathfrak{s l}_{3}$-modules, and of $B_{3}$ on their zero weight spaces are generically irreducible, a fact which refines a result proved by Kwon $[\mathrm{Kw}]$ in the context of quantum Weyl groups, and to which we shall return below. For $\mathfrak{g} \not$ $\mathfrak{s l}_{2}, \mathfrak{s l}_{3}$, the situation is radically different and we prove

Theorem 1.4. If $\mathfrak{g} ¥ \mathfrak{s l}_{2}, \mathfrak{s l}_{3}$, there exists a simple $\mathfrak{g}$-module $V$ the zero weight space of which is reducible under the joint action of $\mathcal{C}_{\mathfrak{g}}$ and of $W$. In particular, $\mathcal{C}_{\mathfrak{g}}$ and $Z(U \mathfrak{g})$ do not generate $U \mathfrak{g}^{\mathfrak{h}}$.

For $\mathfrak{g} ¥ \mathfrak{s l}_{n}$, our $V$ is in fact the kernel of the commutator map $[\cdot, \cdot]: \mathfrak{g} \wedge \mathfrak{g} \rightarrow$ $\mathfrak{g}$. For $\mathfrak{g} \cong \mathfrak{s l}_{n}, \operatorname{Ker}([\cdot, \cdot])$ is reducible and the construction of a suitable $V$ relies on the following general reducibility criterion, valid for any $\mathfrak{g}$. Let $V$ be a simple $\mathfrak{g}$-module with zero weight space $V[0] \neq\{0\}$. If $V$ is selfdual, it is acted on by a linear involution $\Theta_{V}$ such that, for any $X \in \mathfrak{g}$, $\Theta_{V} X \Theta_{V}^{-1}=\Theta(X)$ where $\Theta$ is the Chevalley involution of $\mathfrak{g}$ relative to a given choice of simple root vectors. Since $\Theta$ acts as -1 on $\mathfrak{h}$ and fixes the Casimirs $\kappa_{\alpha}$ and $\widetilde{W}, \Theta_{V}$ leaves $V[0]$ invariant and commutes with $\mathcal{C}_{\mathfrak{g}}$ and $\widetilde{W} . V[0]$ is therefore reducible under $\mathcal{C}_{\mathfrak{g}}$ and $\widetilde{W}$ whenever $\Theta_{V}$ does not act as a scalar on it.

To prove that this is the case for some $V$, we note further that if $\mathfrak{r} \subset \mathfrak{g}$ is a reductive subalgebra normalised by $\Theta$ and $V$ is such that its restriction to $\mathfrak{r}$ contains a zero-weight vector $u$ lying in a simple $\mathfrak{r}$-summand $U$ which is not self-dual, then $\Theta_{V} u$ cannot be proportional to $u$ since $U \cap \Theta_{V} U=\{0\}$. To summarise, our initial problem reduces to finding simple, self-dual $\mathfrak{g}-$ modules $V$ whose restriction to some reductive subalgebra $\mathfrak{r} \subset \mathfrak{g}$ contains non-self dual summands intersecting $V[0]$ non-trivially. For $\mathfrak{g}=\mathfrak{s l}_{n}$, we 
construct such $V$ 's by using the Gelfand-Zetlin branching rules for the inclusion $\mathfrak{g l}_{n-1} \subset \mathfrak{g l}_{n}[\mathrm{GZ}]$.

In section 5, we use our results to disprove a conjecture of Kwon and Lusztig on quantum Weyl group actions of the braid group $B_{n}[\mathrm{Kw}]$. To state it, recall that the Drinfeld-Jimbo quantum group $U_{\hbar} \mathfrak{g}$ corresponding to $\mathfrak{g}$ defines, on any of its integrable representations $\mathcal{V}$, an action of the braid group $B_{\mathfrak{g}}$ called the quantum Weyl group action, which is a deformation of the action of $\widetilde{W}$ on the $\mathfrak{g}$-module $V=\mathcal{V} / \hbar \mathcal{V}$ [Lu, KR, So]. In [Kw], Kwon considered the case of $\mathfrak{g}=\mathfrak{s l}_{n}$ and gave a necessary condition for the zero weight space of $\mathcal{V}$ to be irreducible under $B_{\mathfrak{g}}=B_{n}$. He showed in particular that the zero weight spaces of all $U_{\hbar \mathfrak{s l}}$-modules are irreducible under $B_{3}$. Based on these findings he and Lusztig conjectured that this should hold for all $B_{n}, n \geq 4$.

Theorem 1.5. The Kwon-Lusztig conjecture is false for any simple, complex Lie algebra $\mathfrak{g} ¥ \mathfrak{s l}_{2}, \mathfrak{s l}_{3}$.

Our disproof is based on the simple observation that the quantum Chevalley involution $\Theta_{\hbar}$ of $U_{\hbar \mathfrak{g}}$ acts on any self-dual $U_{\hbar} \mathfrak{g}$-module $\mathcal{V}$ and that its restriction to the zero weight space $\mathcal{V}[0]$ centralises the action of $B_{\mathfrak{g}}$. We then remark that $\Theta_{\hbar}$ acts as a scalar on $\mathcal{V}[0]$ iff the classical Chevalley involution acts as a scalar on the zero weight space of the $\mathfrak{g}$-module $V=\mathcal{V} / \hbar \mathcal{V}$ and rely on the results of section 4 .

In section 6 we show that, despite the reducibility results of $\S 4$, the connection $\nabla_{\kappa}$ yields irreducible monodromy representations of $B_{\mathfrak{g}}$ of arbitrarily large dimensions. For $\mathfrak{g} \cong \mathfrak{s l}_{n}$, we show in fact that the weight spaces of all Cartan powers of the adjoint representation are irreducible under the the Casimirs $\kappa_{\alpha}$.

In section 7 we show that, when $\mathfrak{g}$ is isomorphic to $\mathfrak{s l}_{n}, n \geq 4$, or $\mathfrak{g}_{2}$, the zero weight space of most self-dual, simple $\mathfrak{g}$-modules is reducible under the Casimir algebra $\mathcal{C}_{\mathfrak{g}}$ of $\mathfrak{g}$, thus strengthening the results of section 4. More precisely, let $V$ be a non-trivial, simple, self-dual $\mathfrak{g}$-module with zero weight space $V[0] \neq\{0\}$. Then, we obtain the following classification results

Theorem 1.6. If $\mathfrak{g} \cong \mathfrak{s l}_{n}, n \geq 4, V[0]$ is irreducible under $\mathcal{C}_{\mathfrak{g}}$ if, and only if its highest weight $\lambda \in \mathbb{Z}^{n}$ is of one of the following forms

(i) $\lambda=(p, 0, \ldots, 0,-p), p \in \mathbb{N}$.

(ii) $\lambda=(\underbrace{1, \ldots, 1}_{k}, 0, \ldots, 0, \underbrace{-1, \ldots,-1}_{k}), 0 \leq k \leq n / 2$.

(iii) $\lambda=(p, p,-p,-p), p \in \mathbb{N}$.

Theorem 1.7. If $\mathfrak{g} \cong \mathfrak{g}_{2}, V[0]$ is irreducible under $\mathcal{C}_{\mathfrak{g}}$ if, and only if $V$ is fundamental representation or its second Cartan power. 
Partial classification results pointing to the same phenomenon are obtained in [HMTL] for $\mathfrak{g}=\mathfrak{s o}_{2 n}, \mathfrak{s o}_{2 n+1}, \mathfrak{s p}_{2 n}$. The proofs of theorems 1.6-1.7 rely on the use of the Chevalley involution $\Theta$ outlined above and branching to the subalgebras $\mathfrak{g l}_{k} \subset \mathfrak{s l}_{n}$ and $\mathfrak{s l}_{3} \subset \mathfrak{g}_{2}$ respectively. They show in fact that $V[0]$ is irreducible under $\mathcal{C}_{\mathfrak{g}}$ if, and only if $\Theta$ acts as a scalar on it. It seems natural to conjecture that this should be so for any $\mathfrak{g}$.

It is interesting to note how the reducibility results of section 7 contrast with the following theorem of Etingof, which is reproduced with his kind permission in section 8 . Let $\beta \in \sum_{i=1}^{n} \mathbb{N} \cdot \alpha_{i}$ be a fixed, non-negative linear combination of simple roots and, for $\mu \in \mathfrak{h}^{*}$, let $M_{\mu}[\mu-\beta]$ be the subspace of weight $\mu-\beta$ of the Verma module of highest weight $\mu$.

Theorem 1.8 (Etingof). There exists a Zariski open set $\mathcal{O}_{\beta} \subset \mathfrak{h}^{*}$ such that, for any $\mu \in \mathcal{O}_{\beta}, M_{\mu}[\mu-\beta]$ is irreducible under the Casimir algebra $\mathcal{C}_{\mathfrak{g}}$.

The above theorem has as corollary the following interesting result, which is also proved in $\S 8$

Theorem 1.9 (Etingof). The centraliser of the Casimir algebra $\mathcal{C}_{\mathfrak{g}}$ in $U \mathfrak{g}$ is generated by $\mathfrak{h}$ and the centre of $U \mathfrak{g}$.

Acknowledgements. We wish to heartily thank Allen Knutson whose observations on reading $[\mathrm{KM}]$ initiated this project. We are also grateful to $\mathrm{R}$. Buchweitz, C. De Concini, P. Etingof, M. Kashiwara, F. Knop, B. Kostant, C. Laskowski, A. Okounkov, C. Procesi, R. Rouquier, S. Yuzvinksy and A. Wassermann for a number of useful discussions and the referees for pointing out a number of inaccuracies and improvements to the exposition in an earlier version of this paper. The research for this project was partly carried out while the first author visited the Institut de Mathématiques de Jussieu in June 2000 and 2001 and while the second author was a post-doctoral fellow at MSRI during the academic year 2000-2001. We are grateful to both institutions for their financial support and pleasant working conditions.

\section{Flat connections on $\mathfrak{h}_{\text {reg }}$}

2.1. The flat connection $\nabla_{\kappa}$. Let $\mathfrak{g}$ be a complex, semi-simple Lie algebra with Cartan subalgebra $\mathfrak{h}$ and root system $R=\{\alpha\} \subset \mathfrak{h}^{*}$. Let

$$
\mathfrak{h}_{\text {reg }}=\mathfrak{h} \backslash \bigcup_{\alpha \in R} \operatorname{Ker}(\alpha)
$$

be the set of regular elements in $\mathfrak{h}$ and $V$ a finite-dimensional $\mathfrak{g}$-module. We shall presently define a flat connection on the trivial vector bundle $\mathfrak{h}_{\text {reg }} \times V$ over $\mathfrak{h}_{\text {reg }}{ }^{2}$. We need for this purpose the following flatness criterion due to Kohno [Ko2]. Let $B$ be a complex, finite-dimensional vector space and $\mathcal{A}=\left\{H_{i}\right\}_{i \in I}$ a finite collection of hyperplanes in $B$ determined by the linear forms $\phi_{i} \in B^{*}, i \in I$.

\footnotetext{
2 all vector bundles and connections considered in this section are holomorphic.
} 
Lemma 2.1. Let $V$ be a finite-dimensional vector space and $\left\{r_{i}\right\} \subset \operatorname{End}(V)$ a family indexed by $I$. Then,

$$
\nabla=d-\sum_{i \in I} \frac{d \phi_{i}}{\phi_{i}} \cdot r_{i}
$$

defines a flat connection on $(B \backslash \mathcal{A}) \times V$ iff, for any subset $J \subseteq I$ maximal for the property that $\bigcap_{j \in J} H_{j}$ is of codimension 2, the following relations hold for any $j \in J$

$$
\left[r_{j}, \sum_{j^{\prime} \in J} r_{j^{\prime}}\right]=0
$$

REMARK. Since the relations (2.2) are homogeneous, a solution $\left\{r_{i}\right\}_{i \in I}$ of (2.2) defines in fact a one-parameter family of representations

$$
\rho_{h}: \pi_{1}(B \backslash \mathcal{A}) \longrightarrow G L(V)
$$

parametrised by $h \in \mathbb{C}$ where $\rho_{h}$ is the monodromy of the connection (2.1) with $r_{i}$ replaced by $h \cdot r_{i}$.

For any $\alpha \in R$, choose root vectors $e_{\alpha} \in \mathfrak{g}_{\alpha}, f_{\alpha} \in \mathfrak{g}_{-\alpha}$ such that $\left[e_{\alpha}, f_{\alpha}\right]=$ $h_{\alpha}=\alpha^{\vee}$ and let

$$
\kappa_{\alpha}=\frac{\langle\alpha, \alpha\rangle}{2}\left(e_{\alpha} f_{\alpha}+f_{\alpha} e_{\alpha}\right) \in U \mathfrak{g}
$$

be the truncated Casimir operator of the three-dimensional subalgebra $\mathfrak{s}_{2}^{\alpha} \subset$ $\mathfrak{g}$ spanned by $e_{\alpha}, h_{\alpha}, f_{\alpha}$ relative to the restriction to $\mathfrak{s l}_{2}^{\alpha}$ of a fixed multiple $\langle\cdot, \cdot\rangle$ of the Killing form of $\mathfrak{g}$. Note that $\kappa_{\alpha}$ does not depend upon the particular choice of $e_{\alpha}$ and $f_{\alpha}$ and that $\kappa_{-\alpha}=\kappa_{\alpha}$. Let $R^{+} \subset R$ be the set of positive roots corresponding to a choice of simple roots $\alpha_{1}, \ldots, \alpha_{n}$ of $\mathfrak{g}$.

Theorem 2.2. The one-form

$$
\nabla_{\kappa}=d-h \sum_{\alpha \in R^{+}} \frac{d \alpha}{\alpha} \cdot \kappa_{\alpha}=d-\frac{h}{2} \sum_{\alpha \in R} \frac{d \alpha}{\alpha} \cdot \kappa_{\alpha}
$$

defines, for any $h \in \mathbb{C}$, a flat connection on $\mathfrak{h}_{\mathrm{reg}} \times V$ which is reducible with respect to the weight space decomposition of $V$.

Proof. By lemma 2.1, we must show that for any rank 2 root subsystem $R_{0} \subseteq R$ determined by the intersection of $R$ with a 2 -dimensional subspace in $\mathfrak{h}^{*}$, the following holds for any $\alpha \in R_{0}^{+}=R_{0} \cap R^{+}$

$$
\left[\kappa_{\alpha}, \sum_{\beta \in R_{0}^{+}} \kappa_{\beta}\right]=0
$$

This may be proved by an explicit computation by considering in turn the cases where $R_{0}$ is of type $A_{1} \times A_{1}, A_{2}, B_{2}$ or $G_{2}$ but is more easily settled by the following elegant observation of A. Knutson [Kn]. Let $\mathfrak{g}_{0} \subseteq \mathfrak{g}$ be the semi-simple Lie algebra with root system $R_{0}, \mathfrak{h}_{0} \subset \mathfrak{h}$ its Cartan subalgebra and $C_{0} \in Z\left(U \mathfrak{g}_{0}\right)$ its Casimir operator. Then, $\sum_{\beta \in R_{0}^{+}} \kappa_{\beta}-C_{0}$ lies in $U \mathfrak{h}_{0}$ so that $(2.5)$ holds since $\kappa_{\alpha}$ commutes with $\mathfrak{h}_{0}$. The reducibility of $\nabla_{\kappa}$ with 
respect to the $\mathfrak{h}$-action on $V$ is an immediate consequence of the fact that the operators $\kappa_{\alpha}$ are of weight zero

REMARK. Altough $V$ admits a hermitian inner product with respect to which the Casimirs $\kappa_{\alpha}$ are self-adjoint, it is easy to check that the connection $\nabla_{\kappa}$ is not unitary with respect to the corresponding constant inner product on $\mathfrak{h}_{\text {reg }} \times V$. However, the fact that the connection $\nabla_{\kappa}$ for $\mathfrak{g}=\mathfrak{s l}_{n}$ coincides with the (genus 0) Knizhnik-Zamolodchikov connection on $n$ points for $\mathfrak{g}^{\prime}=\mathfrak{s l}_{k}$ via Howe duality [TL2, thm. 3.5], and that the latter is conjectured to be unitary on the subbundle of conformal blocks for suitable rational values of $h[\mathrm{Ga}]^{3}$, suggests that the connection $\nabla_{\kappa}$ ought to be unitary for any $\mathfrak{g}$. It is an interesting open problem to determine whether this is so.

Let $W$ be the Weyl group of $\mathfrak{g}$. Fix a basepoint $t_{0} \in \mathfrak{h}_{\text {reg }}$, let $\left[t_{0}\right]$ be its image in $\mathfrak{h}_{\text {reg }} / W$ and let $P_{\mathfrak{g}}=\pi_{1}\left(\mathfrak{h}_{\text {reg }} ; t_{0}\right), B_{\mathfrak{g}}=\pi_{1}\left(\mathfrak{h}_{\text {reg }} / W ;\left[t_{0}\right]\right)$ be the generalised pure and full braid groups of type $\mathfrak{g}$. We adhere here to the convention that for a pointed topological space $\left(X, x_{0}\right)$, the composition $q \cdot p$ of two paths $p, q \in \pi_{1}\left(X ; x_{0}\right)$ is given by $p$ followed by $q$ so that the holonomy of a flat vector bundle $(\mathcal{V}, \nabla)$ at $x_{0}$ is a group homomorphism $\pi\left(X ; x_{0}\right) \rightarrow G L\left(\mathcal{V}_{x_{0}}\right)$. The fibration $\mathfrak{h}_{\text {reg }} \rightarrow \mathfrak{h}_{\text {reg }} / W$ gives rise to the exact sequence

$$
1 \longrightarrow P_{\mathfrak{g}} \longrightarrow B_{\mathfrak{g}} \longrightarrow W \longrightarrow 1
$$

where the rightmost arrow is obtained by associating to $p \in B_{\mathfrak{g}}$ the unique $w \in W$ such that $w^{-1} t_{0}=\widetilde{p}(1)$ where $\widetilde{p}$ is the unique lift of $p$ to a path in $\mathfrak{h}_{\text {reg }}$ such that $\widetilde{p}(0)=t_{0}$.

By theorem 2.2, the monodromy of $\nabla_{\kappa}$ yields a one-parameter family of representations of $P_{\mathfrak{g}}$ on $V$ preserving its weight space decomposition. We wish to extend this action to one of $B_{\mathfrak{g}}$, by pushing $\nabla_{\kappa}$ down to a flat connection on the quotient $\mathfrak{h}_{\text {reg }} / W$. Since $W$ does not act on $V$, this requires choosing an action of $B_{\mathfrak{g}}$ on $V$. Let for this purpose $G$ be the complex, connected and simply-connected Lie group with Lie algebra $\mathfrak{g}, T$ its torus with Lie algebra $\mathfrak{h}$ and $N(T) \subset G$ the normaliser of $T$ so that $W \cong N(T) / T$. We regard $B_{\mathfrak{g}}$ as acting on $V$ by choosing a homomorphism $\sigma: B_{\mathfrak{g}} \rightarrow N(T)$ compatible with

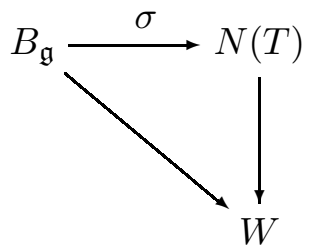

Such $\sigma$ 's abound and we describe in $\S 2.5$ a class of them which we call Tits extensions [Ti]. Let $\widetilde{\mathfrak{h}_{\text {reg }}} \stackrel{p}{\rightarrow} \mathfrak{h}_{\text {reg }}$ be the universal cover of $\mathfrak{h}_{\text {reg }}$ and $\mathfrak{h}_{\text {reg }} / W$.

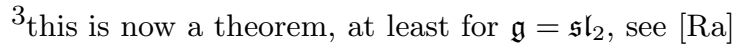


Proposition 2.3. The one-form $p^{*} \nabla_{\kappa}$ defines a $B_{\mathfrak{g}}$-equivariant flat connection on $p^{*}\left(\mathfrak{h}_{\text {reg }} \times V\right)=\widetilde{\mathfrak{h}_{\text {reg }}} \times V$. It therefore descends to a flat connection on the vector bundle

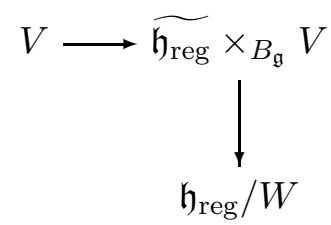

which is reducible with respect to the weight space decomposition of $V$.

Proof. $B_{\mathfrak{g}}$ acts on $\Omega^{\bullet}\left(\widetilde{\mathfrak{h}_{\text {reg }}}, V\right)=\Omega^{\bullet}\left(\widetilde{\mathfrak{h}_{\text {reg }}}\right) \otimes V$ by $\gamma \rightarrow\left(\gamma^{-1}\right)^{*} \otimes \sigma(\gamma)$. Thus, if $\gamma \in B_{\mathfrak{g}}$ projects onto $w \in W$, we get using $p \cdot \gamma^{-1}=w^{-1} \cdot p$,

$$
\gamma p^{*} \nabla_{\kappa} \gamma^{-1}=d-\frac{h}{2} \sum_{\alpha \in R} d p^{*} w \alpha / p^{*} w \alpha \otimes \sigma(\gamma) \kappa_{\alpha} \sigma(\gamma)^{-1}
$$

Since $\kappa_{\alpha}$ is independent of the choice of the root vectors $e_{\alpha}, f_{\alpha}$ in (2.3), $\operatorname{Ad}(\sigma(\gamma)) \kappa_{\alpha}=\kappa_{w \alpha}$ and the above is equal to $p^{*} \nabla_{\kappa}$ as claimed. $p^{*} \nabla_{\kappa}$ is flat and commutes with the fibrewise action of $\mathfrak{h}$ by theorem 2.2

Thus, for any homomorphism $\sigma: B_{\mathfrak{g}} \rightarrow N(T)$ compatible with (2.6), proposition 2.3 yields a one-parameter family of monodromy representations

$$
\rho_{h}^{\sigma}: B_{\mathfrak{g}} \longrightarrow G L(V)
$$

which permutes the weight spaces of $V$ compatibly with the action of $W$ on $\mathfrak{h}^{*}$. By standard ODE theory, $\rho_{h}^{\sigma}$ depends analytically on the complex parameter $h$ and, when $h=0$, is equal to the action of $B_{\mathfrak{g}}$ on $V$ given by $\sigma$. We record for later use the following elementary

Proposition 2.4. Let $\gamma \in B_{\mathfrak{g}}=\pi_{1}\left(\mathfrak{h}_{\mathrm{reg}} / W ;\left[t_{0}\right]\right)$ and let $\widetilde{\gamma}:[0,1] \rightarrow \mathfrak{h}_{\mathrm{reg}}$ be a lift of $\gamma$. Then,

$$
\rho_{h}^{\sigma}(\gamma)=\sigma(\gamma) \mathcal{P}(\widetilde{\gamma})
$$

where $\mathcal{P}(\widetilde{\gamma}) \in G L(V)$ is the parallel transport along $\widetilde{\gamma}$ for the connection $\nabla_{\kappa}$ on $\mathfrak{h}_{\text {reg }} \times V$.

Proof. Let $\widetilde{\widetilde{\gamma}}:[0,1] \rightarrow \widetilde{\mathfrak{h}_{\text {reg }}}$ be a lift of $\gamma$ and $\widetilde{\gamma}$ so that $\widetilde{\widetilde{\gamma}}(1)=\gamma^{-1} \widetilde{\widetilde{\gamma}}(0)$. Then, since the connection on $p^{*}\left(\mathfrak{h}_{\text {reg }} \times V\right)$ is the pull-back of $\nabla_{\kappa}$, and that on $\left(p^{*}\left(\mathfrak{h}_{\text {reg }} \times V\right)\right) / B_{\mathfrak{g}}$ the quotient of $p^{*} \nabla_{\kappa}$, we find that

$$
\rho_{h}^{\sigma}(\gamma)=\mathcal{P}(\gamma)=\sigma(\gamma) \mathcal{P}(\widetilde{\gamma})=\sigma(\gamma) \mathcal{P}(\widetilde{\gamma})
$$

REMARK. By (2.7), the representation $\rho_{h}^{\sigma}$ depends on the choice of the homomorphism $\sigma$ satisfying (2.6). However, the representations corresponding to Tits extensions are all equivalent since these are conjugate by elements of $T$ (see $\S 2.5$ ). Note also that the restriction of $\rho_{h}^{\sigma}$ to the zero weight space of $V$ does not depend on $\sigma$ since $W \cong N(T) / T$ acts canonically on it. 
REMARK. Note that, by (2.7), the restriction of $\rho_{h}^{\sigma}$ to the pure braid group $P_{\mathfrak{g}}$ does not coincide with the monodromy of the connection $\nabla_{\kappa}$. Rather, it differs from it by the $T$-valued character given by the restriction of $\sigma$ to $P_{\mathfrak{g}}$.

REMARK. By Brieskorn's theorem, $B_{\mathfrak{g}}$ is presented on generators $S_{1}, \ldots, S_{n}$ labelled by the simple simple reflections $s_{1}, \ldots, s_{n} \in W$ with relations

$$
\underbrace{S_{i} S_{j} \cdots}_{m_{i j}}=\underbrace{S_{j} S_{i} \cdots}_{m_{i j}}
$$

for any $1 \leq i<j \leq n$ where the number $m_{i j}$ of factors on each side is equal to the order of $s_{i} s_{j}$ in $W[\mathrm{Br}]$. Each $S_{i}$ may be obtained as a small loop in $\mathfrak{h}_{\text {reg }} / W$ around the reflecting hyperplane $\operatorname{Ker}\left(\alpha_{i}\right)$ of $s_{i}$.

2.2. Variants of $\nabla_{\kappa}$. If $p_{\alpha} \in U \mathfrak{h}, \alpha \in R$, is a collection of polynomials in $\mathfrak{h}$, the connection

$$
d-\frac{h}{2} \sum_{\alpha \in R} \frac{d \alpha}{\alpha} \cdot\left(\kappa_{\alpha}+p_{\alpha}\right)
$$

is flat by theorem 2.2 since $\left[\kappa_{\alpha}, p_{\beta}\right]=\left[p_{\alpha}, p_{\beta}\right]=0$ for any $\alpha, \beta \in R$. It is moreover $W$-equivariant if, in addition, $w p_{\alpha}=p_{w \alpha}$ for any $w \in W$. The corresponding monodromy representation of $P_{\mathfrak{g}}$ is equal to that of the connection $\nabla_{\kappa}$ tensored with the character $\chi: P_{\mathfrak{g}} \rightarrow T$ given by the monodromy of the abelian connection

$$
d-\frac{h}{2} \sum_{\alpha \in R} \frac{d \alpha}{\alpha} \cdot p_{\alpha}
$$

and therefore does not significantly differ from the monodromy of $\nabla_{\kappa}$. A possible choice is to set $p_{\alpha}=\langle\alpha, \alpha\rangle / 2 \cdot h_{\alpha}^{2}$ which yields the connection

$$
\nabla_{C}=d-h \sum_{\alpha \in R^{+}} \frac{d \alpha}{\alpha} \cdot C_{\alpha}
$$

where $C_{\alpha} \in U \mathfrak{s l}_{2}^{\alpha}$ is the full Casimir operator of $\mathfrak{s i}_{2}^{\alpha}$.

2.3. The holonomy Lie algebra $\mathfrak{a}(\mathcal{A})$. Kohno's lemma 2.1 gives a description of the holonomy Lie algebra $\mathfrak{a}(\mathcal{A})$ of a general hyperplane arrangement $\mathcal{A}=\left\{\mathcal{H}_{i}\right\}_{i \in I}$ as the quotient of the free Lie algebra on generators $\left\{r_{i}\right\}_{i \in I}$ by the relations (2.2). When $\mathcal{A}=\mathcal{A}_{\mathfrak{g}}=\{\operatorname{Ker}(\alpha)\}_{\alpha \in R}$ is the arrangement of root hyperplanes of $\mathfrak{g}$, theorem 2.2 is equivalent to the fact that the assignment $r_{\alpha} \rightarrow \kappa_{\alpha}$ extends to an algebra homomorphism

$$
\phi: U \mathfrak{a}\left(\mathcal{A}_{\mathfrak{g}}\right) \longrightarrow U \mathfrak{g}
$$

of the universal enveloping algebra of $\mathfrak{a}\left(\mathcal{A}_{\mathfrak{g}}\right)$ to that of $\mathfrak{g}$ satisfying

$$
\phi\left(U \mathfrak{a}\left(\mathcal{A}_{\mathfrak{g}}\right)_{m}\right) \subset U \mathfrak{g}_{2 m}
$$

for any $m \in \mathbb{N}$, where the superscript denotes the degree corresponding to the natural filtrations on both algebras. We simply note here the following 
Proposition 2.5. If one of the simple factors of $\mathfrak{g}$ is not isomorphic to $\mathfrak{s l}_{2}$, the map $\phi: U \mathfrak{a}\left(\mathcal{A}_{\mathfrak{g}}\right) \longrightarrow U \mathfrak{g}$ is not injective.

Proof. The following argument was pointed out to us by R. Buchweitz. It suffices to show that, if $\mathfrak{g} ¥ \mathfrak{s l}_{2}, \mathfrak{a}\left(\mathcal{A}_{\mathfrak{g}}\right)$ contains a free Lie algebra on at least two generators, for then $U \mathfrak{a}\left(\mathcal{A}_{\mathfrak{g}}\right)$ has exponential growth with respect to its filtration, whereas $U \mathfrak{g}$, being isomorphic to $S \mathfrak{g}$, only grows polynomially. Let $A_{1} \times A_{1} ¥ R_{0} \subseteq R$ be a rank two root subsystem with positive roots $\beta_{1}, \ldots, \beta_{p}, p \geq 3$. Let $\mathcal{F}_{p-1}$ be the free Lie algebra on generators $x_{1}, \ldots, x_{p-1}$ and consider the maps

$$
\mathcal{F}_{p-1} \stackrel{i}{\rightarrow} \mathfrak{a}\left(\mathcal{A}_{\mathfrak{g}}\right) \stackrel{\pi}{\rightarrow} \mathcal{F}_{p-1}
$$

given by $i\left(x_{j}\right)=r_{\beta_{j}}, j=1 \ldots p-1$ and

$$
\pi\left(r_{\alpha}\right)=\left\{\begin{array}{cl}
0 & \text { if } \alpha \notin R_{0} \\
x_{j} & \text { if } \alpha=\beta_{j}, \text { with } 1 \leq j \leq p-1 \\
-\sum_{j=1}^{p-1} x_{j} & \text { if } \alpha=\beta_{p}
\end{array}\right.
$$

It is easy to see that $\pi$ is well-defined, so that $\pi \circ i=\mathrm{id}$ and $i$ gives an embedding of $\mathcal{F}_{p-1}$ into $\mathfrak{a}\left(\mathcal{A}_{\mathfrak{g}}\right)$

REMARK. It seems an interesting problem to find a generating set of relations for the kernel of the map $\phi$ above. One such relation may be obtained for any rank two root subsystem $R_{2} \subset R$ such that the intersection of its $\mathbb{Z}$ span with $R$ is equal to $R_{2}$ but the intersection of its $\mathbb{R}$-span with $R$ strictly contains $R_{2}$. This is the case for the root system of type $A_{2}$ given by the long roots in the root system of type $\mathfrak{g}_{2}$ or for the root system of type $A_{1} \times A_{1}$ generated by any pair of long roots in the root system of type $C_{n}$. One then has $\left[\kappa_{\alpha}, \sum_{\beta \in R_{2}} \kappa_{\beta}\right]=0$ in $U \mathfrak{g}$, but $\left[r_{\alpha}, \sum_{\beta \in R_{2}} r_{\beta}\right] \neq 0$ in $\mathfrak{a}\left(\mathcal{A}_{\mathfrak{g}}\right)$.

2.4. Triviality of $\widetilde{\mathfrak{h}_{\text {reg }}} \times_{P_{\mathfrak{g}}} V$. We show below that the pull-back to $\mathfrak{h}_{\text {reg }}$ of the vector bundle $\widetilde{\mathfrak{h}_{\text {reg }}} \times_{B_{\mathfrak{g}}} V$ constructed in proposition 2.3 , namely

$$
\mathcal{V}=\widetilde{\mathfrak{h}_{\text {reg }}} \times{ }_{P_{\mathfrak{g}}} V
$$

is trivial ${ }^{4}$. Our trivialisation of $\mathcal{V}$ yields an action of $W$ on $\mathfrak{h}_{\mathrm{reg}} \times V$ by a one-cocycle, that is by $w(t, v)=(w t, A(w, t) v)$ where $A(w, t) \in G L(V)$ satisfies

$$
A\left(w_{1} w_{2}, t\right)=A\left(w_{1}, w_{2} t\right) A\left(w_{2}, t\right)
$$

which we compute explicitly. These results will not be used elsewhere in the paper.

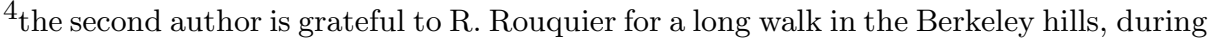
which we took turns in convincing each other that the bundle was trivial, then non-trivial, then trivial again, until sheer exhaustion and the late hour of the night suspended, but did not resolve, the argument.
} 
Let $\sigma: B_{\mathfrak{g}} \rightarrow N(T)$ be a homomorphism making (2.6) commute. The restriction of $\sigma$ to the pure braid group $P_{\mathfrak{g}}$ maps into $T$ and therefore factors through the abelianisation of $P_{\mathfrak{g}}$. The following gives an explicit description of the latter as a $W=B_{\mathfrak{g}} / P_{\mathfrak{g}}-$ module.

Proposition 2.6. Let $Z$ be the free abelian group with one generator $\gamma_{\alpha}$ for each positive root $\alpha$ of $\mathfrak{g}$ and let $W$ act on $Z$ by $w \gamma_{\alpha}=\gamma_{|w \alpha|}$, where $|w \alpha|$ is equal to $\pm w \alpha$ according to whether $w \alpha$ is positive or negative. Then,

(i) the assignment $\gamma_{\alpha_{i}} \rightarrow S_{i}^{2}$ extends uniquely to a $W$-equivariant isomorphism $Z \cong P_{\mathfrak{g}} /\left[P_{\mathfrak{g}}, P_{\mathfrak{g}}\right]$.

(ii) Under the Hurewicz isomorphism $P_{\mathfrak{g}} /\left[P_{\mathfrak{g}}, P_{\mathfrak{g}}\right] \cong H_{1}\left(\mathfrak{h}_{\text {reg }}, \mathbb{Z}\right), \gamma_{\alpha}$ is mapped onto a positively oriented simple loop around the hyperplane $\operatorname{Ker}(\alpha)$.

Proof. (i) is proved in [Ti, Thm. 2.5]. (ii) under the Hurewicz isomorphism, the action of $W$ on $P_{\mathfrak{g}} /\left[P_{\mathfrak{g}}, P_{\mathfrak{g}}\right]$ coincides with its natural geometric action on $H_{1}\left(\mathfrak{h}_{\text {reg }}, \mathbb{Z}\right)$. By Brieskorn's description of the generators of $B_{\mathfrak{g}}$, $\gamma_{\alpha_{i}}=S_{i}^{2}$ is mapped onto a positively oriented simple loop around the hyperplane $\operatorname{Ker}\left(\alpha_{i}\right)$ so that (ii) follows by $W$-equivariance

For any positive root $\alpha$, pick an element $\lambda_{\alpha} \in \mathfrak{h}$ such that $\exp \left(2 \pi i \lambda_{\alpha}\right)=$ $\sigma\left(\gamma_{\alpha}\right)$ and consider the flat connection on $\mathfrak{h}_{\text {reg }} \times V$ given by

$$
\nabla_{\sigma}=d-\sum_{\alpha \in R^{+}} \frac{d \alpha}{\alpha} \cdot \lambda_{\alpha}
$$

Fix a basepoint $t_{0} \in \mathfrak{h}_{\text {reg }}$ and identify $\widetilde{\mathfrak{h}_{\text {reg }}}$ with the space of paths in $\mathfrak{h}_{\text {reg }}$ pinned at $t_{0}$, modulo homotopy equivalence. Denote by $\mathcal{P}_{\sigma}(p) \in T$ parallel transport with respect to $\nabla_{\sigma}$ along one such path $p$. Then,

\section{Proposition 2.7.}

(i) The map $\widetilde{\mathfrak{h}_{\text {reg }}} \times V \rightarrow \mathfrak{h}_{\text {reg }} \times V$ given by

$$
(p, v) \rightarrow\left(p(1), \mathcal{P}_{\sigma}(p) v\right)
$$

descends to an isomorphism of vector bundles $\iota: \widetilde{\mathfrak{h}_{\text {reg }}} \times_{P_{\mathfrak{g}}} V \cong$ $\mathfrak{h}_{\text {reg }} \times V$.

(ii) The right action of $B_{\mathfrak{g}}$ on $\widetilde{\mathfrak{h}_{\mathrm{reg}}} \times V$ descends, via $\iota$, to one of $W$ on $\mathfrak{h}_{\text {reg }} \times V$ given by

$$
w(t, v)=\left(w^{-1} t, \mathcal{P}_{\sigma}\left(w^{-1} p_{t}\right) \mathcal{P}_{\sigma}(\widetilde{\gamma}) \sigma(\gamma)^{-1} \mathcal{P}_{\sigma}\left(p_{t}\right)^{-1} v\right)
$$

where $p_{t}$ is any pinned path in $\mathfrak{h}_{\text {reg }}$ with $p_{t}(1)=t, \gamma \in B_{\mathfrak{g}}$ is any element with image $w$ and $\widetilde{\gamma}$ is its lift to a path in $\mathfrak{h}_{\text {reg }}$ with $\widetilde{\gamma}(0)=t_{0}$.

ProOF. One readily checks, by using proposition 2.6 , that the monodromy $P_{\mathfrak{g}} \rightarrow T$ of $\nabla_{\sigma}$ coincides with the restriction of $\sigma$ to $P_{\mathfrak{g}}$ from which (i) follows at once. (ii) is a simple computation 
2.5. Tits extensions. Let $\sigma: B_{\mathfrak{g}} \rightarrow N(T)$ be a homomorphism making the diagram (2.6) commute. Tits has given a simple construction of a canonical, but not exhaustive, class of such $\sigma$ which differ from each other via conjugation by an element of $T$. We summarise below the properties of this class obtained in [Ti]. For any simple root $\alpha_{i}, i=1 \ldots n$, let $S L_{2}(\mathbb{C}) \cong G_{i} \subseteq G$ be the subgroup with Lie algebra spanned by $e_{\alpha_{i}}, f_{\alpha_{i}}, h_{\alpha_{i}}, T_{i}=\exp \left(\mathbb{C} \cdot h_{\alpha_{i}}\right) \subset G_{i}$ its torus and $N_{i}$ the normaliser of $T_{i}$ in $G_{i}$. Denote by $s_{i} \in W$ the orthogonal reflection corresponding to $\alpha_{i}$.

\section{Proposition 2.8.}

(i) For any choice of $\sigma_{i} \in N_{i} \backslash T_{i}, i=1 \ldots n$, the assignment $S_{i} \rightarrow \sigma_{i}$ extends uniquely to a homomorphism $\sigma: B_{\mathfrak{g}} \rightarrow N(T)$ making (2.6) commute.

(ii) If $\sigma, \sigma^{\prime}: B_{\mathfrak{g}} \rightarrow N(T)$ are the homomorphisms corresponding to the choices $\left\{\sigma_{i}\right\}_{i=1}^{n}$ and $\left\{\sigma_{i}^{\prime}\right\}_{i=1}^{n}$ respectively, there exists $t \in T$ such that, for any $S \in B_{\mathfrak{g}}$

$$
\sigma(S)=t \sigma^{\prime}(S) t^{-1}
$$

(iii) For any such $\sigma: B_{\mathfrak{g}} \rightarrow N(T)$, the subgroup $\sigma\left(B_{\mathfrak{g}}\right) \subset N(T)$ is an extension of $W$ by $\mathbb{Z}_{2}^{n}$ canonically isomorphic to the group generated by the symbols $a_{i}, i=1 \ldots n$ subject to the relations

$$
\begin{aligned}
\underbrace{a_{i} a_{j} \cdots}_{m_{i j}} & =\underbrace{a_{j} a_{i} \cdots}_{m_{i j}} \\
a_{i}^{2} a_{j}^{2} & =a_{j}^{2} a_{i}^{2} \\
a_{i}^{4} & =1 \\
a_{i} a_{j}^{2} a_{i}^{-1} & =a_{j}^{2} a_{i}^{-2\left\langle\alpha_{i}^{\vee}, \alpha_{j}\right\rangle}
\end{aligned}
$$

for any $1 \leq i \neq j \leq n$, where the number $m_{i j}$ of factors on each side of (2.9) is equal to the order of $s_{i} s_{j}$ in $W$. The isomorphism is given by sending $a_{i}$ to $\sigma_{i}$.

Proof. (i) We must show that the $\sigma_{i}$ satisfy the braid relations (2.8). For any $1 \leq i \neq j \leq n$, set $s_{i j}=s_{i} s_{j} \cdots \in W$ and $\sigma_{i j}=\sigma_{i} \sigma_{j} \cdots \in N(T)$ where each product has $m_{i j}-1$ factors. The braid relations in $W$ may be written as $s_{i j} s_{j^{\prime}}=s_{j} s_{i j}$ where $j^{\prime}=j$ or $i$ according to whether $m_{i j}$ is even or odd. Thus, $s_{i j}^{-1} s_{j} s_{i j}=s_{j^{\prime}}$ and therefore,

$$
\delta_{i j}=\sigma_{j^{\prime}}^{-1} \sigma_{i j}^{-1} \sigma_{j} \sigma_{i j} \in T \cap\left(\sigma_{j^{\prime}}^{-1} \sigma_{i j}^{-1} N_{j} \sigma_{i j}\right)=T \cap \sigma_{j^{\prime}}^{-1} N_{j^{\prime}}=T_{j^{\prime}}
$$

Repeating the argument with $i$ and $j$ permuted, we find that $\delta_{j i} \in T_{i^{\prime}}$ with $i^{\prime}=i$ or $j$ according to whether $m_{i j}$ is even or odd. Thus, $\delta_{i j}=$ $\delta_{j i}^{-1} \in T_{i^{\prime}} \cap T_{j^{\prime}}=\{1\}$ where the latter assertion follows from the simple connectedness of $G$, and the $\sigma_{i}$ satisfy (2.8). 
(ii) Let $t_{i} \in T_{i}$ be such that $\sigma_{i}=\sigma_{i}^{\prime} t_{i}$ and choose $c_{i} \in \mathbb{C}$ such that $t_{i}=$ $\exp \left(c_{i} h_{\alpha_{i}}\right)$. Since

$$
\left(s_{i}-1\right) \sum_{j=1}^{n} c_{j} \lambda_{j}^{\vee}=-c_{i} h_{\alpha_{i}}
$$

where the $\lambda_{i}^{\vee} \in \mathfrak{h}$ are the fundamental coweights defined by $\alpha_{i}\left(\lambda_{j}^{\vee}\right)=\delta_{i j}$, we find

$$
\exp \left(-\sum_{j} c_{j} \lambda_{j}^{\vee}\right) \sigma_{i}^{\prime} \exp \left(\sum_{j} c_{j} \lambda_{j}^{\vee}\right)=\sigma_{i}^{\prime} \exp \left(c_{i} h_{\alpha_{i}}\right)=\sigma_{i}
$$

so that $\sigma$ and $\sigma^{\prime}$ are conjugate.

(iii) The $\sigma_{i}$ satisfy (2.9)-(2.12) since $x_{j}^{2}=\exp \left(i \pi \alpha_{j}^{\vee}\right)$ for any $x_{j} \in N_{j} \backslash T_{j}$. Let $K_{\sigma} \cong \mathbb{Z}_{2}^{n}$ be the group generated by the $\sigma_{i}^{2}$ and $K_{\sigma} \subset \bar{K}_{\sigma} \subset \sigma\left(B_{\mathfrak{g}}\right)$ the kernel of the projection $\sigma\left(B_{\mathfrak{g}}\right) \rightarrow W$. By (2.12), $K_{\sigma}$ is a normal subgroup of $\sigma\left(B_{\mathfrak{g}}\right)$ and $\sigma\left(B_{\mathfrak{g}}\right) / K_{\sigma}$ is generated by the images $\overline{\sigma_{i}}$ of $\sigma_{i}$ which, in addition to the braid relations satisfy $\bar{\sigma}_{i}^{2}=1$. Thus, $\sigma\left(B_{\mathfrak{g}}\right) / K_{\sigma}$ is a quotient of $W$, $K_{\sigma}=\bar{K}_{\sigma}$ and $\sigma\left(B_{\mathfrak{g}}\right) / K_{\sigma} \cong W$. The same argument shows that if $\Gamma$ is the abstract group generated by $a_{1}, \ldots, a_{n}$ subject to $(2.9)-(2.12)$, and $A \subset \Gamma$ is the subgroup generated by the $a_{i}^{2}$, then $\Gamma / A \cong W \cong \sigma\left(B_{\mathfrak{g}}\right) / K_{\sigma}$. But $A$ is a quotient of $\mathbb{Z}_{n}^{2}$ so that the canonical surjection of $\Gamma$ onto $\sigma\left(B_{\mathfrak{g}}\right)$ is an isomorphism of $A$ onto $K_{\sigma}$ and therefore an isomorphism of $\Gamma$ onto $\sigma\left(B_{\mathfrak{g}}\right)$

We shall henceforth only use homomorphisms $\sigma: B_{\mathfrak{g}} \rightarrow N(T)$ of the form given by proposition 2.8 and refer to them, or their image $\widetilde{W}=\sigma\left(B_{\mathfrak{g}}\right) \subset$ $N(T)$ as Tits extensions of $W$. Note that, given a choice of simple root vectors $e_{\alpha_{i}}, f_{\alpha_{i}}, i=1 \ldots n$, any element of $N_{i} \backslash T_{i}$ is necessarily of the form

$$
\begin{aligned}
\sigma_{i}\left(t_{i}\right) & =\exp \left(t_{i} e_{\alpha_{i}}\right) \exp \left(-t_{i}^{-1} f_{\alpha_{i}}\right) \exp \left(t_{i} e_{\alpha_{i}}\right) \\
& =\exp \left(-t_{i}^{-1} f_{\alpha_{i}}\right) \exp \left(t_{i} e_{\alpha_{i}}\right) \exp \left(-t_{i}^{-1} f_{\alpha_{i}}\right)
\end{aligned}
$$

for a unique $t_{i} \in \mathbb{C}^{*}$ so that a Tits extension may be given by choosing elements $t_{1}, \ldots, t_{n} \in \mathbb{C}^{*}$.

\section{GeneriC IRREDUCibiLity of MONODROMY REPRESENTATIONS}

3.1. In this section, we study in detail the reducibility of the monodromy of a flat connection of the form (2.1), namely

$$
\nabla=d-h \sum_{i \in I} \frac{d \phi_{i}}{\phi_{i}} \cdot r_{i}
$$

where the residues $r_{i}$ act on the finite-dimensional vector space $V$ and are assumed to satisfy the relations (2.2). Let

$$
\rho_{h}: \pi_{1}(B \backslash \mathcal{A}) \longrightarrow G L(V)
$$

be the corresponding one-parameter family of monodromy representations. If $V$ is reducible under the $r_{i}, \rho_{h}$ is clearly reducible for all values of $h$. We 
wish to prove a converse statement. To formulate it, we need the following

DEFINITION. An analytic curve $\rho_{h}$ of representations of a finitely-generated group $\Gamma$ is generically irreducible if $\rho_{h}$ is irreducible for all $h$ in the complement of an analytic set.

The main result of this section is the following

Theorem 3.1. If $V$ is irreducible under the $r_{i}$, the monodromy representation $\rho_{h}$ is generically irreducible.

The proof of theorem 3.1 occupies the rest of this section; in $\S 3.5$, we apply this result to the monodromy of the connection $\nabla_{\kappa}$. It proceeds by noting that, because reducibility is a closed condition, an analytic curve $\rho_{h}$ of representations is either generically irreducible or reducible for all $h$. In the latter case, we prove the existence of an analytic, multivalued curve germ $U\left(h^{1 / m}\right)$ of proper subspaces of $V$ invariant under $\rho_{h}$. A simple calculation then shows that the subspace $\left.U\left(h^{1 / m}\right)\right|_{h^{1 / m}=0} \subsetneq V$ is invariant under the $r_{i}$. Note that in general, a single-valued germ of invariant subspaces may fail to exist. For example, the curve $c(h)$ of reducible representations of $\Gamma=\mathbb{Z}$ given by

$$
c(h)=\left(\begin{array}{ll}
1 & 1 \\
h & 1
\end{array}\right)
$$

with $h \in \mathbb{C} \backslash\{ \pm 1\}$, only admits the multivalued family of eigenlines $(1, \pm \sqrt{h})$. We will see that such branching is the worst behaviour that can occur.

3.2. The variety of reducible representations. Let $\Gamma$ be a finitelygenerated group. The set of representations $\operatorname{Hom}(\Gamma, G L(V))$ has the structure of an affine variety defined over $\mathbb{Q}$. Indeed, if $\left\{\gamma_{1}, \ldots, \gamma_{r}\right\}$ is a system of generators of $\Gamma, \operatorname{Hom}(\Gamma, G L(V)) \subset G L(V)^{r}$ is the subset of $r$-tuples of elements satisfying the relations which define $\Gamma$.

Proposition 3.2. The set $\operatorname{Hom}^{\mathrm{red}}(\Gamma, G L(V))$ of reducible representations is a Zariski closed subset of $\operatorname{Hom}(\Gamma, G L(V))$.

Proof. Let $\operatorname{Gr}_{p}(V)$ be the Grassmannian of $p$-planes in $V$ and set

$$
\mathcal{R}_{p}(\Gamma)=\left\{(\rho, U) \in \operatorname{Hom}(\Gamma, G L(V)) \times \operatorname{Gr}_{p}(V) \mid \rho(\Gamma) U=U\right\}
$$

We claim that $\mathcal{R}_{p}(\Gamma)$ is a Zariski closed subset of $\operatorname{Hom}(\Gamma, G L(V)) \times \operatorname{Gr}_{p}(V)$. Indeed, regarding $U \in \operatorname{Gr}_{p}(V)$ as a decomposable $p$-tensor $\Lambda=u_{1} \wedge \cdots \wedge u_{p} \in$ $\mathbb{P}\left(\bigwedge^{p} V\right)$ via the Plücker embedding, we see that the invariance of $U$ under $\rho \in \operatorname{Hom}(\Gamma, G L(V))$ is equivalent to the relations

$$
\Lambda \wedge \rho\left(\gamma_{i}\right) \Lambda=0
$$

for all $i=1 \ldots r$, where $\wedge$ is the exterior multiplication in $\bigwedge^{*}\left(\bigwedge^{p} V\right)$. Since the projection $p_{1}: \operatorname{Hom}(\Gamma, G L(V)) \times \operatorname{Gr}_{p}(V) \rightarrow \operatorname{Hom}(\Gamma, G L(V))$ is closed 
[Mu, pg. 76], the set

$$
\begin{aligned}
\operatorname{Hom}^{\mathrm{red}, p}(\Gamma, G L(V)) & =\left\{\rho \in \operatorname{Hom}(\Gamma, G L(V)) \mid \exists U \in \operatorname{Gr}_{p}(V), \rho U=U\right\} \\
& =p_{1}\left(\mathcal{R}_{p}(\Gamma)\right)
\end{aligned}
$$

is a closed subset of $\operatorname{Hom}(\Gamma, G L(V))$ and therefore so is

$$
\operatorname{Hom}^{\mathrm{red}}(\Gamma, G L(V))=\bigcup_{p=1}^{\operatorname{dim} V-1} \operatorname{Hom}^{\mathrm{red}, p}(\Gamma, G L(V))
$$

Corollary 3.3. An analytic curve $\rho_{h}: \Gamma \longrightarrow G L(V)$ of representations is either generically irreducible or reducible for all values of $h$.

REMARK. The variety $\mathcal{R}^{p}(\Gamma)$ is clearly defined over $\mathbb{Q}$. By [St, prop. 1.1.1], this is also true of $\operatorname{Hom}^{\text {red,p}}(\Gamma, G L(V))$ since it is defined over $\overline{\mathbb{Q}}$ by $[\mathrm{Mu}$, pg. 76] and the underlying set of $\overline{\mathbb{Q}}$-points is stable under the action of $\operatorname{Gal}(\overline{\mathbb{Q}}, \mathbb{Q})$. Finally, the regular map

$$
\pi_{p}: \mathcal{R}_{p}(\Gamma) \longrightarrow \operatorname{Hom}^{\text {red, } p}(\Gamma, G L(V))
$$

induced by projection on the first factor is also defined over $\mathbb{Q}$ since $p_{1}$ is.

3.3. The existence of a multivalued section. Retain the notation of $\S 3.2$. We will be concerned in this subsection with constructing a multivalued section to the projection $\pi_{p}$ over a curve germ in $\operatorname{Hom}^{\text {red, } p}(\Gamma, G L(V))$. Let $\Gamma_{r}$ be the free group $\Gamma_{r}$ on $r$ generators. The commutative diagram

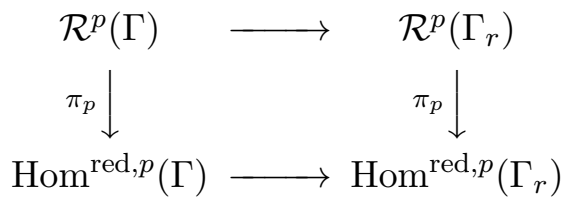

shows that it suffices to find such a section for $\Gamma_{r}$. For the remainder of this subsection we therefore assume that $\Gamma=\Gamma_{r}$.

We shall in fact prove the existence of a formal multivalued section. The same argument yields an analytic multivalued one. We need some notation. Let $A$ be the coordinate ring of $\operatorname{Hom}(\Gamma, G L(V)) \cong G L(V)^{r}, \mathfrak{a}_{p}, \mathfrak{a} \subset A$ the ideals of $\operatorname{Hom}^{\text {red, } p}(\Gamma, G L(V))$, $\operatorname{Hom}^{\text {red }}(\Gamma, G L(V))$ respectively and $S_{p}=$ $A / \mathfrak{a}_{p}, S=A / \mathfrak{a}$ their coordinate rings. Let $\widetilde{G r}_{p}(V)$ be the affine cone in $\bigwedge^{p}(V)$ defined by the Plücker equations, $B_{p}=\mathbb{C}\left[\operatorname{Hom}(\Gamma, G L(V)) \times \widetilde{\operatorname{Gr}}_{p}(V)\right]$ and $\mathfrak{b}_{p} \subset B_{p}$ the ideal defined by equations (3.2). Set

$$
R_{p}=B_{p} / \mathfrak{b}_{p}=\mathbb{C}\left[\widetilde{\mathcal{R}}^{p}(\Gamma)\right]
$$

where $\widetilde{\mathcal{R}}^{p}(\Gamma) \subset \operatorname{Hom}(\Gamma, G L(V)) \times \widetilde{\mathrm{Gr}}_{p}(V)$ is the preimage of the variety $\mathcal{R}_{p}(\Gamma)$ defined by (3.1). The projection $\pi_{p}$ induces a ring homomorphism $\psi_{p}: S_{p} \rightarrow R_{p}$. 
Let $\rho$ be a fixed reducible representation. A formal curve of representations starting at $\rho$ is a homomorphism of $\mathbb{C}$-algebras

$$
\phi: A \longrightarrow \mathbb{C}[[h]]
$$

where $\mathbb{C}[[h]]$ is the ring of formal power series in $h$ whose reduction mod $h$ is the $\mathbb{C}$-point $\rho . \phi$ is a curve of reducible representations if it descends to $S$. Let $\mathfrak{p}$ be the kernel of $\phi . \mathfrak{p}$ is a prime ideal since $\mathbb{C}[[h]]$ is an integral domain and $\mathfrak{p} \supset \mathfrak{a}=\bigcap_{p=1}^{n-1} \mathfrak{a}_{p}$ so that $\mathfrak{p} \supset \mathfrak{a}_{p}$ for some $p$ and we obtain a homomorphism $\phi: S_{p} \longrightarrow \mathbb{C}[[h]]$.

Definition. A multivalued formal section of $\pi_{p}$ is a homomorphism $\chi$ fitting into the following commutative diagram

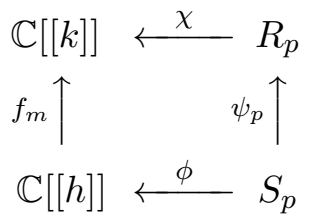

where $f_{m}(h)=k^{m}$.

Let $\mathcal{F}=\mathbb{C}((h))$ be the field of fractions of $\mathbb{C}[[h]], \overline{\mathcal{F}}$ its algebraic closure and $\iota: \mathcal{F} \rightarrow \overline{\mathcal{F}}$ the corresponding inclusion. The key step in finding a formal multivalued section is the following

Proposition 3.4. There exists a homomorphism of $\mathbb{C}$-algebras $\tau: R_{p} \rightarrow \overline{\mathcal{F}}$ such that the following diagram is commutative

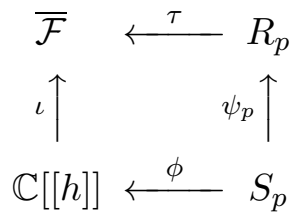

and $\tau\left(x_{I}\right) \neq 0$ for some $I$, where $x_{I}$ are the Plücker coordinates on $\widetilde{\operatorname{Gr}}_{p}(V)$.

Proof. Note first that $\overline{\mathcal{F}}$ is isomorphic to $\mathbb{C}$ since both are algebraically closed fields of uncountable transcendence degree over $\mathbb{Q}$, and choose an isomorphism $\phi: \mathbb{C} \rightarrow \overline{\mathcal{F}}$. Since $X=\operatorname{Hom}^{\text {red, } p}(\Gamma, G L(V)), Y=\widetilde{\mathcal{R}}_{p}$ are defined over $\mathbb{Q}, \phi$ induces an isomorphism between the the $\mathbb{C}$ and $\overline{\mathcal{F}}$-points of $X$ (resp. $Y$ ) which we denote by $\underline{X}(\mathbb{C}), \underline{X}(\overline{\mathcal{F}})$ (resp. $\underline{Y}(\mathbb{C}), \underline{Y}(\overline{\mathcal{F}})$ ). Since the projection $\pi_{p}$ is defined over $\mathbb{Q}$, we therefore get a commutative diagram

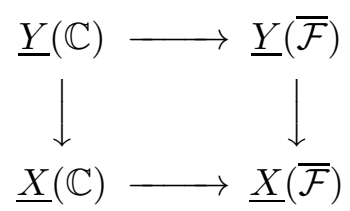

and the rightmost vertical arrow is onto 
We now construct the desired multivalued formal section. Recall that the algebraic closure of $\mathbb{C}((h))$ is the field $\mathbb{C}\left(\left(h^{\frac{1}{\infty}}\right)\right)$ obtained by adjoining all the roots of $h$ to $\mathbb{C}((h))$ [Wal, thm. 3.1].

Theorem 3.5. There exists an $m \geq 1$ and a homomorphism of $\mathbb{C}$-algebras $\chi: \widehat{R}_{p} \rightarrow \mathbb{C}[[k]]$ such that the following diagram is commutative

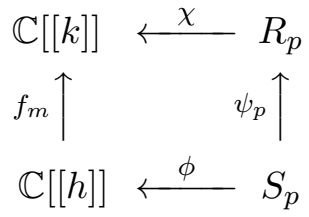

and $\chi\left(x_{I}\right) \neq 0$ for some $I$.

Proof. Proposition 3.4 yields a homomorphism $\tau$ taking values in the quotient field $\mathbb{C}((k))$ of $\mathbb{C}[[k]]$ for some root $k$ of $h$. Such a homomorphism amounts to assigning an element of $\mathbb{C}((k))$ to each of the generators $g_{i j}^{k}$, $1 \leq i, j \leq \operatorname{dim}(V), 1 \leq k \leq r$ and $x_{I}$ of $R_{p}$ in such a way that these elements satisfy the defining equations of $\widetilde{\mathcal{R}}_{p}(\Gamma)$. By $(3.3), \tau\left(g_{i j}^{k}\right)=\iota \phi\left(g_{i j}^{k}\right) \in \mathbb{C}[[k]]$. Since the equations are homogeneous in the $x_{I}$, we can multiple the $\tau\left(x_{I}\right)$ by an appropriate power of $k$ so that the resulting elements of $\mathbb{C}((k))$ are in $\mathbb{C}[[k]]$

The following result explains the meaning of the $\mathbb{C}[[k]]$-point $\chi$.

Proposition 3.6. The homomorphism $\chi$ gives rise to a rank $p$ summand $\mathcal{U}$ of the module $V[[k]]=V \otimes \mathbb{C}[[k]]$ together with an $r$-tuple of invertible (over $\mathbb{C}[[k]])$ matrices $g^{1}\left(k^{m}\right), \ldots, g^{r}\left(k^{m}\right)$ leaving $\mathcal{U}$ invariant.

3.4. Proof of theorem 3.1. Let $\Gamma$ be the fundamental group $\pi_{1}(B \backslash \mathcal{A})$. It is well-known that $\Gamma$ is finitely-generated, see e.g., [BMR, prop. A2, pg. 181]. If the curve $\rho_{h}$ of monodromy representations is not generically irreducible, it lies, by corollary 3.3 , in the variety of reducible representations and therefore in some $\operatorname{Hom}^{\text {red, } p}(\Gamma, G L(V)), 1 \leq p \leq \operatorname{dim} V-1$. Denote by $\mathbb{C}\{k\}$ the ring of convergent power series at $k=0$. Let $\chi$ be a multivalued analytic section and let $\mathcal{U}$ be the corresponding rank $p$ summand of the free $\mathbb{C}\{k\}$-module $\mathcal{V}=V \otimes \mathbb{C}\{k\}$ obtained by applying the analytic version of proposition 3.6. $\mathcal{U}$ corresponds to an multivalued analytic curve $U(k)$ in $\operatorname{Gr}_{p}(V)$ which is invariant under $\rho_{h}$.

Set $\widetilde{\rho}_{k}=\rho_{k^{m}}$ and let $\widetilde{\rho}$ be the element of $\operatorname{Hom}(\Gamma, G L(\mathcal{V}))$ corresponding to $\widetilde{\rho}_{k}$. Since $\widetilde{\rho}_{k}$ is an analytic function of $h=k^{m}$, the elements

$$
A(\gamma)=\frac{I-\widetilde{\rho}(\gamma)}{k^{m}}
$$

leave $\mathcal{V}$ invariant. It follows that $A(\gamma) \mathcal{U} \subset \mathcal{U}$ since $\widetilde{\rho}(\Gamma) \mathcal{U}=\mathcal{U}$. The following standard result shows that the subspace $U(0) \subsetneq V$ is invariant under the residues $r_{i}$, thus concluding the proof of theorem 3.1 
Lemma 3.7. For each hyperplane $H_{j}$ of the arrangement $\mathcal{A}$, there exists an element $\gamma_{j} \in \pi_{1}(B \backslash \mathcal{A})$ such that

$$
\rho_{h}\left(\gamma_{j}\right)=1+2 \pi i h \cdot r_{j} \bmod h^{2}
$$

Proof. Let $x_{0} \in B \backslash \mathcal{A}$ be a base point and $\gamma_{j} \in \pi_{1}\left(B \backslash \mathcal{A} ; x_{0}\right)$ a generator of monodromy around $H_{j}$ (see, e.g., [BMR, pg. 180-1]). Recall that such an element is obtained as follows. Choose a path $p:[0,1] \rightarrow B$ such that

$$
p(0)=x_{0}, \quad p\left(\left[0,1[) \subset B \backslash \mathcal{A} \text { and } \quad p(1) \in H_{j} \backslash \bigcup_{j^{\prime} \neq j} H_{j^{\prime}}\right.\right.
$$

Let $D$ be a small ball centred at $p(1)$ and contained in $B \backslash \bigcup_{j^{\prime} \neq j} H_{j^{\prime}}$, let $u \in[0,1[$ be such that $p(s) \in D$ for any $s \geq u$ and let $\ell$ be a positively oriented generator of $\pi_{1}\left(D \backslash H_{j} ; p(u)\right) \cong \mathbb{Z}$. Then,

$$
\gamma_{i}=p_{u}^{-1} \cdot \ell \cdot p_{u}
$$

where $p_{u}(t)=p(u t)$ and the concatenation of paths is read from right to left. Picard iteration readily yields that, $\bmod h^{2}$,

$$
\rho_{h}\left(\gamma_{j}\right)=1+h \sum_{i \in I} \int_{\gamma_{j}} \frac{d \phi_{i}}{\phi_{i}} \cdot r_{i}=1+h \sum_{i \in I} \int_{\ell} \frac{d \phi_{i}}{\phi_{i}} \cdot r_{i}=1+2 \pi i h \cdot r_{j}
$$

where the last equality follows from the residue theorem since the forms $d \phi_{j^{\prime}} / \phi_{j^{\prime}}, j^{\prime} \neq j$ do not have any poles in $D$

The above proof readily yields the following analogue of theorem 3.1 in the formal setting. Let

$$
\widetilde{\rho}: \pi_{1}(B \backslash \mathcal{A}) \longrightarrow G L(V((h)))
$$

be the representation obtained by regarding $\rho_{h}$ as formal in $h$, letting $\pi_{1}(B \backslash$ $\mathcal{A})$ act on $V[[h]]$ and extending coefficients to $V((h))$. Then

Theorem 3.8. If $V$ is irreducible under the $r_{i}, \widetilde{\rho}$ is absolutely irreducible, that is irreducible over the algebraic closure $\mathbb{C}\left(\left(h^{\frac{1}{\infty}}\right)\right)$ of $\mathbb{C}((h))$.

3.5. Generic irreducibility of the monodromy of $\nabla_{\kappa}$. Assume now that $V$ is a $\mathfrak{g}$-module and let $\widetilde{W}=\sigma\left(B_{\mathfrak{g}}\right)$ be a Tits extension with sign group $\Sigma=\sigma\left(P_{\mathfrak{g}}\right) \cong \mathbb{Z}_{2}^{n}$. Let

$$
\rho_{h}^{\sigma}: B_{\mathfrak{g}} \longrightarrow G L(V)
$$

be the corresponding one-parameter family of monodromy representations defined by proposition 2.3 and $\rho^{\sigma}: B_{\mathfrak{g}} \longrightarrow G L(V((h)))$ the representation obtained by regarding $\rho_{h}^{\sigma}$ as formal in $h$ and extending coefficients to $\mathbb{C}((h))$.

Theorem 3.9. Let $U \subseteq V$ be a subspace invariant, and irreducible under the Casimirs $\kappa_{\alpha}$ and $\widetilde{W}$. Then,

(i) $\rho_{h}^{\sigma}: B_{\mathfrak{g}} \longrightarrow G L(U)$ is generically irreducible.

(ii) $\rho^{\sigma}: B_{\mathfrak{g}} \longrightarrow G L(U((h)))$ is absolutely irreducible. 
Proof. (i) Assume $\rho_{h}^{\sigma}$ to be reducible for all $h$. Proceeding as in the proof of theorem 3.1, we find a $1 \leq p \leq \operatorname{dim} U-1$ and a rank $p$-summand $\mathcal{X}$ of $\mathcal{U}=U \otimes \mathbb{C}\{k\}$ invariant under the germ of $\rho_{h}^{\sigma}$ at $h=0$. In particular, $\mathcal{X}(0)$ is invariant under $\rho_{0}^{\sigma}\left(B_{\mathfrak{g}}\right)=\widetilde{W}$. For any positive root $\alpha$, let $\gamma_{\alpha} \in P_{\mathfrak{g}}$ be the generator of monodromy around the hyperplane $\operatorname{Ker}(\alpha)$ given by lemma 3.7. Note that $\sigma\left(\gamma_{\alpha}\right)$ lies in the sign group $\Sigma$ and therefore has order 1 or 2 . By lemma 3.7 and proposition 2.4 we find that, $\bmod h^{2}$,

$$
\rho_{h}^{\sigma}\left(\gamma_{\alpha}^{2}\right)=\sigma\left(\gamma_{\alpha}\right)^{2}\left(1+2 \pi i h \cdot \kappa_{\alpha}\right)^{2}=1+4 \pi i h \cdot \kappa_{\alpha}
$$

so that $\mathcal{X}(0)$ is also invariant under the Casimirs $\kappa_{\alpha}$. The proof of (ii) is identical

Similarly, we obtain

Theorem 3.10. Let $U \subseteq V$ be a subspace invariant, and irreducible under the Casimirs $\kappa_{\alpha}$ and $\Sigma$. Then,

(i) $\rho_{h}^{\sigma}: P_{\mathfrak{g}} \longrightarrow G L(U)$ is generically irreducible.

(ii) $\rho^{\sigma}: P_{\mathfrak{g}} \longrightarrow G L(U((h)))$ is absolutely irreducible.

We specialise our results further to the case where $U$ is the zero weight space $V[0]$ of $V$. Recall that the latter is canonically acted upon by $W \cong N(T) / T$ so that the restriction of $\rho_{h}^{\sigma}$ to $V[0]$ does not depend upon the choice of $\sigma$. We owe the following somewhat surprising observation to B. Kostant

Proposition 3.11. $V[0]$ is irreducible under the Casimirs $\kappa_{\alpha}$ iff it is irreducible under the $\kappa_{\alpha}$ and $W$.

Proof. The simple reflection $s_{i} \in W$ acts on the zero weight space $V_{n}^{i}[0]$ of the irreducible $\mathfrak{s l}_{2}^{\alpha_{i}}$-module of dimension $2 n+1$ as multiplication by $(-1)^{n}$. Thus, if $p_{\varepsilon}^{i}, \varepsilon=0,1$ are the spectral projections for the restriction of $C_{\alpha_{i}}$ to $V[0]$ corresponding to the Casimir eigenvalues of $V_{n}^{i}$, with $n=\epsilon \bmod 2, s_{i}$ acts on $V[0]$ as $p_{0}^{i}-p_{1}^{i}$ and is therefore a polynomial in $C_{\alpha_{i}}$. It follows that a subspace $U \subseteq V[0]$ invariant under the $\kappa_{\alpha}$ is also invariant under $W$

Corollary 3.12. The following statements are equivalent

(i) $V[0]$ is irreducible under the Casimirs $\kappa_{\alpha}$.

(ii) $V[0]$ is generically irreducible under $P_{\mathfrak{g}}$.

(iii) $V[0]((h))$ is absolutely irreducible under $P_{\mathfrak{g}}$.

(iv) $V[0]$ is generically irreducible under $B_{\mathfrak{g}}$.

(v) $V[0]((h))$ is absolutely irreducible under $B_{\mathfrak{g}}$.

\section{The Casimir algebra $\mathcal{C}_{\mathfrak{g}}$ OF $\mathfrak{g}$}

Recall from the Introduction that the Casimir algebra $\mathcal{C}_{\mathfrak{g}}$ of $\mathfrak{g}$ is the algebra

$$
\mathcal{C}_{\mathfrak{g}}=\left\langle\kappa_{\alpha}\right\rangle_{\alpha \in R_{+}} \vee \mathfrak{h} \subseteq U \mathfrak{g}^{\mathfrak{h}}
$$

generated by the $\kappa_{\alpha}$, or equivalently the Casimirs $C_{\alpha}$, and $\mathfrak{h}$ inside $U \mathfrak{g}^{\mathfrak{h}}$. 


\subsection{The Casimir algebra of $\mathfrak{s l}_{3}$.}

Theorem 4.1. If $\mathfrak{g}=\mathfrak{s l}_{3}$, then

(i) $\mathcal{C}_{\mathfrak{g}}$ is a proper subalgebra of $U \mathfrak{g}^{\mathfrak{h}}$.

(ii) $U \mathfrak{g}^{\mathfrak{h}}$ is generated by $\mathcal{C}_{\mathfrak{g}}$ and the center of $U \mathfrak{g}$. In particular, the Casimirs $\kappa_{\alpha}$ act irreducibly on the weight spaces of simple $\mathfrak{g}$-modules.

We need some preliminary results. Let $e_{1}, \ldots, e_{n}$ be the canonical basis of $\mathbb{C}^{n}$ and $E_{i j} e_{k}=\delta_{j k} e_{i}$ the corresponding elementary matrices. For any sequence $I=\left(i_{1}, \ldots, i_{k}\right)$ of distinct elements of $\{1, \ldots, n\}$, set

$$
E_{I}=E_{i_{1} i_{2}} E_{i_{2} i_{3}} \cdots E_{i_{k-1} i_{k}} E_{i_{k} i_{1}} \in U \mathfrak{s i}_{n}^{\mathfrak{h}}
$$

Proposition 4.2. If $\mathfrak{g}=\mathfrak{s l}_{n}, U \mathfrak{g}^{\mathfrak{h}}$ is generated as an algebra by $\mathfrak{h}$ and the monomials $E_{I}$ corresponding to sequences $\left(i_{1}, \ldots, i_{k}\right)$ such that $i_{1}=\min _{l} i_{l}$.

Proof. It suffices to show that the $E_{I}$ and $\mathfrak{h}$ generate $\operatorname{gr}\left(U \mathfrak{g}^{\mathfrak{h}}\right)=S \mathfrak{g}^{\mathfrak{h}}$. $S \mathfrak{g}^{\mathfrak{h}}$ is clearly spanned by elements of the form $p \cdot E_{I, \sigma}$ where $p \in S \mathfrak{h}, I=$ $\left(i_{1}, \ldots, i_{k}\right)$ is a sequence of elements in $\{1, \ldots, n\}, \sigma \in \mathfrak{S}_{k}$ is a permutation and $E_{I, \sigma}=E_{i_{1} i_{\sigma(1)}} \cdots E_{i_{k} i_{\sigma(k)}}$. Writing $\sigma$ as a product $\tau_{1} \cdots \tau_{r}$ of disjoint cycles with $\tau_{j}=\left(m_{j}^{1} \cdots m_{j}^{k_{j}}\right)$ and setting $I_{j}=\left(i_{m_{j}^{1}}, \cdots, i_{m_{j}}^{k_{j}}\right)$ shows that, in $S \mathfrak{g}, E_{I, \sigma}=E_{I_{1}} \cdots E_{I_{r}}$

Corollary 4.3. If $\mathfrak{g}=\mathfrak{s l}_{3}, U \mathfrak{g}^{\mathfrak{h}}$ is generated by $\mathfrak{h}$,

$$
F_{12}=E_{12} E_{21} \quad F_{13}=E_{13} E_{31} \quad F_{23}=E_{23} E_{32}
$$

and

$$
G_{123}=E_{12} E_{23} E_{31} \quad G_{132}=E_{13} E_{32} E_{21}
$$

Proof of Theorem 4.1. (ii) Let $\theta_{i}-\theta_{j}, 1 \leq i<j \leq 3$ be the positive roots of $\mathfrak{g}$. The $\mathfrak{s l}_{2}$-triple corresponding to $\theta_{i}-\theta_{j}$ is $\left\{E_{i j}, E_{j i}, E_{i i}-E_{j j}\right\}$ and

$$
\kappa_{\theta_{i}-\theta_{j}}=E_{i j} E_{j i}+E_{j i} E_{i j}=2 F_{i j} \bmod \mathfrak{h}
$$

$S \mathfrak{g}^{\mathfrak{h}}$ is therefore generated, as a $\operatorname{Gr}\left(\mathcal{C}_{\mathfrak{g}}\right)$-algebra, by $G=G_{123}+G_{132}$ since

$$
G_{123}-G_{132}=F_{13}+\left[F_{23}, F_{12}\right] \in \mathcal{C}_{\mathfrak{g}}
$$

It therefore suffices to show that $G$ lies in the algebra generated by $\mathcal{C}_{\mathfrak{g}}$ and $Z(U \mathfrak{g})$, which is a simple exercise. (i) Let $\sigma$ be the involution of $\mathfrak{g}$ given by $\sigma(X)=-X^{t}$. $\sigma$ leaves $\mathfrak{h}$ invariant and descends to an involution of $Q=U \mathfrak{g}^{\mathfrak{h}} / U \mathfrak{g}^{\mathfrak{h}} \mathfrak{h}$ fixing $\mathcal{C}_{\mathfrak{g}}$. It therefore suffices to show that $\sigma$ does not act trivially on $Q$ or the associated graded $\operatorname{gr}(Q)$. However the image of $G=G_{123}+G_{132}$ in $\operatorname{gr}(Q)$ satisfies $\sigma(G)=-G$ since $\sigma\left(G_{123}\right)=-G_{132}$ in $\operatorname{gr}(Q)$

Corollary 4.4. If $V$ be a simple $\mathfrak{s l}_{3}$-module, the monodromy of $\nabla_{\kappa}$ yields generically irreducible representations of Artin's pure braid group $P_{3}$ on the weight spaces of $V$ and of the braid group $B_{3}$ on the zero weight space of $V$. 
4.2. The Casimir algebra of $\mathfrak{g} \not \mathfrak{s l}_{n}$. Assume that $\mathfrak{g}$ is simple and not isomorphic to $\mathfrak{s l}_{n}$ and let $V$ be the kernel of the commutator map $[\cdot, \cdot]$ : $\mathfrak{g} \wedge \mathfrak{g} \rightarrow \mathfrak{g}$. It is known that $V$ is a simple $\mathfrak{g}$-module [Re].

Theorem 4.5. The zero weight space $V[0]$ is reducible under $\mathcal{C}_{\mathfrak{g}}$. In particular, $\mathcal{C}_{\mathfrak{g}}$ and $Z(U \mathfrak{g})$ do not generate $U \mathfrak{g}^{\mathfrak{h}}$.

Proof. Since $\mathfrak{g} \wedge \mathfrak{g} \cong \mathfrak{g} \oplus V$ and the zero weight space of $\mathfrak{g} \wedge \mathfrak{g}$ has a basis given by $h_{i} \wedge h_{j}, 1 \leq i<j \leq n$, and $e_{\alpha} \wedge f_{\alpha}, \alpha \in R_{+}$, where $h_{1}, \ldots, h_{n}$ is a basis of $\mathfrak{h}$, we find that

$$
\operatorname{dim} V[0]=\frac{n(n-1)}{2}+\left|R_{+}\right|=\frac{n(n-1)}{2}+\frac{m-3 n}{2}
$$

where $m=\operatorname{dim}(\mathfrak{g})>3 n$. Thus, $\mathfrak{h} \wedge \mathfrak{h}$ is a proper subspace of the zero weight space of $V$ and it suffices to show that it is invariant under the $\kappa_{\alpha}$. This follows at once from the fact that, for any $t_{1}, t_{2} \in \mathfrak{h}$,

$$
\begin{aligned}
e_{\alpha} f_{\alpha} t_{1} \wedge t_{2} & =e_{\alpha}\left(\alpha\left(t_{1}\right) f_{\alpha} \wedge t_{2}+\alpha\left(t_{2}\right) t_{1} \wedge f_{\alpha}\right) \\
& =\alpha\left(t_{1}\right) h_{\alpha} \wedge t_{2}+\alpha\left(t_{2}\right) t_{1} \wedge h_{\alpha}
\end{aligned}
$$

4.3. A general reducibility criterion for $V[0]$. Let $\Theta$ be the Chevalley involution of $\mathfrak{g}$ relative to a choice of simple root vectors $e_{\alpha_{i}}, f_{\alpha_{i}}$, i.e., the automorphism of $\mathfrak{g}$ defined by

$$
\Theta\left(e_{\alpha_{i}}\right)=-f_{\alpha_{i}}, \quad \Theta\left(f_{\alpha_{i}}\right)=-e_{\alpha_{i}} \quad \text { and } \quad \Theta\left(h_{\alpha_{i}}\right)=-h_{\alpha_{i}}
$$

If $V$ is a simple, finite-dimensional $\mathfrak{g}$-module, and $V^{\Theta}$ is the module obtained by twisting the action of $\mathfrak{g}$ by $\Theta$, then $V^{\Theta}$ is isomorphic to the dual $V^{*}$ of $V$. In particular, if $V$ is self-dual, there exists an involution $\Theta_{V}$ acting on $V$ such that, for any $X \in \mathfrak{g}$,

$$
\Theta_{V} X \Theta_{V}=\Theta(X)
$$

Although $\Theta_{V}$ is only unique up to a sign, we shall abusively refer to it as the Chevalley involution of $V$. Since $\Theta$ acts as -1 on the Cartan subalgebra $\mathfrak{h}$ and fixes the Casimirs $\kappa_{\alpha}, \Theta_{V}$ leaves the zero weight space $V[0]$ invariant and commutes with the action of $\mathcal{C}_{\mathfrak{g}}$. The following gives a useful criterion to show that $\Theta_{V}$ does not act as a scalar on $V[0]$ and therefore that the latter is reducible under $\mathcal{C}_{\mathfrak{g}}$.

Proposition 4.6. Let $V$ be a self-dual $\mathfrak{g}$-module with $V[0] \neq 0$. Let $\mathfrak{r} \subset \mathfrak{g}$ be a reductive subalgebra normalised by $\mathfrak{h}$. Assume that there exists a nonzero vector $v \in V[0]$ lying in a simple $\mathfrak{r}$-module $U$ such that $U \nsubseteq U^{*}$. Then, $\Theta_{V}$ does not act as a scalar on $V[0]$ and the latter is reducible under $\mathcal{C}_{\mathfrak{g}}$.

Proof. The assumptions imply that $\Theta$ leaves $\mathfrak{r}$ invariant and therefore acts as a Chevalley involution on it. Thus, $\Theta_{V} U \subset V$ is a simple $\mathfrak{r}$-module isomorphic to $U^{*}$ which has zero intersection with $U$ since $U \supsetneqq U^{*}$. In particular, $\Theta_{V} v$ is not proportional to $v$ 
We record for later use the following alternative proof of theorem 4.5.

Proposition 4.7. Let $\mathfrak{g} ¥ \mathfrak{s l}_{n}$ and let $V$ be the simple, self-dual $\mathfrak{g}$-module $V=\operatorname{Ker}[\cdot, \cdot] \subset \bigwedge^{2} \mathfrak{g}$. Then, the Chevalley involution $\Theta$ does not act as a scalar on $V[0]$.

Proof. $\Theta$ acts as +1 on the subspace $\mathfrak{h} \wedge \mathfrak{h} \subset V[0]$ and as -1 on the span of the vectors $e_{\alpha} \wedge f_{\alpha} \in \bigwedge^{2} \mathfrak{g}[0]$. The conclusion follows since, as noted in the proof of theorem $4.5, \mathfrak{h} \wedge \mathfrak{h}$ is a proper subspace of $V[0]$

4.4. The Gelfand-Zetlin branching rules. Let $e_{1}, \ldots, e_{n}$ be the canonical basis of $\mathbb{C}^{n}$ and $E_{a b} e_{c}=\delta_{b c} e_{a}$ the corresponding elementary matrices. Consider the chain of subalgebras

$$
\mathfrak{g l}_{1} \subset \mathfrak{g l}_{2} \subset \cdots \subset \mathfrak{g l}_{n-1} \subset \mathfrak{g l}_{n}
$$

where each $\mathfrak{g l}_{k}$ is spanned by the matrices $E_{i j}, 1 \leq i, j \leq k$. By the GelfandZetlin branching rules [GZ, Zh1], the irreducible representation $V_{\lambda}$ of $\mathfrak{g l}_{k}$ with highest weight $\lambda=\left(\lambda_{1}, \ldots, \lambda_{k}\right) \in \mathbb{Z}^{k}$ decomposes under $\mathfrak{g l}_{k-1}$ as

$$
\operatorname{res}_{\mathfrak{g l}_{k}}^{\mathfrak{g l}_{k-1}} V_{\lambda}=\bigoplus_{\bar{\lambda}} V_{\bar{\lambda}}
$$

where $V_{\bar{\lambda}}$ is the irreducible $\mathfrak{g l}_{k-1}-$ module with highest weight $\bar{\lambda}$ and $\bar{\lambda}=$ $\left(\bar{\lambda}_{1}, \ldots, \bar{\lambda}_{k-1}\right) \in \mathbb{Z}^{k-1}$ ranges over all dominant weights of $\mathfrak{g l}_{k-1}$ satisfying the inequalities

$$
\lambda_{1} \geq \bar{\lambda}_{1} \geq \lambda_{2} \geq \cdots \geq \bar{\lambda}_{k-1} \geq \lambda_{k}
$$

which we denote by $\lambda \succ \bar{\lambda}$. Since the above decomposition is multiplicityfree, it follows, by restricting in stages from $\mathfrak{g l}_{n}$ to $\mathfrak{g l}_{1}$ along (4.1), that any simple $\mathfrak{g l}_{n}$-module $V$ possesses a basis labelled by Gelfand-Zetlin patterns, i.e., arrays $\mu$ of the form

$$
\begin{array}{cccc}
\mu_{1}^{(n)} \mu_{\mu_{1}^{(n-1)}} \mu_{2}^{(n)} & \ldots & \mu_{n-1}^{(n)}{ }_{\mu_{n-1}^{(n-1)}} \mu_{n}^{(n)} \\
& & \ldots & \\
& \mu_{1}^{(2)}{ }_{\mu_{1}^{(1)}} \mu_{2}^{(2)} & \\
& &
\end{array}
$$

where the top row $\mu^{(n)}$ is equal to the highest weight of $V$ and each pair $\mu^{(k)} \in \mathbb{Z}^{k}, \mu^{(k-1)} \mathbb{Z}^{k-1}$ of consecutive rows satisfies $\mu^{(k)} \succ \mu^{(k-1)}$. Up to a scalar factor, the vector $v_{\mu}$ corresponding to the above pattern is uniquely determined by the requirement that it tranforms under each $\mathfrak{g l}_{k} \subset \mathfrak{g l}_{n}$ according to the irreducible representation with highest weight $\mu^{(k)}$. In particular, since the central element $\sum_{i=1}^{k} E_{i i} \in \mathfrak{g l}_{k}$ acts in the latter as multiplication by $\left|\mu^{(k)}\right|=\sum_{i=1}^{k} \mu_{i}^{(k)}$, we find that, for any $1 \leq i \leq n$,

$$
E_{i i} v_{\mu}=\left(\left|\mu^{(i)}\right|-\left|\mu^{(i-1)}\right|\right) v_{\mu}
$$


so that $v_{\mu}$ has weight zero for the action of $\mathfrak{s l}_{n}$ iff, for any $1 \leq i \leq n$,

$$
\left|\mu^{(i)}\right|=i\left|\mu^{(1)}\right|=i \mu_{1}^{(1)}
$$

For later use in $\S 6.1$, we shall need the non-vanishing of some of the matrix coefficients for the action of the simple roots vectors of $\mathfrak{g l}_{n}$ in the above basis. This follows from the explicit formulae for the action of all elementary matrices $E_{i j}$ in a suitably normalised Gelfand-Zetlin basis $v_{\mu}$ which may be found in $[\mathrm{GZ}, \mathrm{Zh} 2]$.

Theorem 4.8 (Gelfand-Zetlin). Let $\mu$ be a Gelfand-Zetlin pattern, then, for any $1 \leq i \leq n-1$

$$
E_{i+1} v_{\mu}=\sum_{\mu^{\prime}} c_{\mu, \mu^{\prime}}^{i} v_{\mu^{\prime}}
$$

where the sum ranges over all patterns $\mu^{\prime}$ obtained from $\mu$ by adding 1 to one of the entries of its ith row and the coefficients $c_{\mu, \mu^{\prime}}^{i}$ are non-zero and,

$$
E_{i+1 i} v_{\mu}=\sum_{\mu^{\prime}} \widetilde{c}_{\mu, \mu^{\prime}}^{i} v_{\mu^{\prime}}
$$

where the sum ranges over all patterns $\mu^{\prime}$ obtained from $\mu$ by substracting 1 to one of the entries of its ith row and the coefficients $\widetilde{c}_{\mu, \mu^{\prime}}^{i}$ are non-zero.

Corollary 4.9. Let $\mu$ be a Gelfand-Zetlin pattern, then

$$
E_{i i+1} E_{i+1 i} v_{\mu}=\sum_{\mu^{\prime}} d_{\mu, \mu^{\prime}}^{i} v_{\mu^{\prime}}
$$

where the sum ranges over all patterns $\mu^{\prime}$ differing from $\mu$ by the addition and the substraction of 1 on a pair of (not necessarily distinct) entries of the ith row and $d_{\mu, \mu^{\prime}}^{i} \neq 0$ if $\mu \neq \mu^{\prime}$.

\subsection{The Casimir algebra of $\mathfrak{s l}_{n}, n \geq 4$.}

Theorem 4.10. If $\mathfrak{g}=\mathfrak{s l}_{n}, n \geq 4$, there exists a simple, self-dual $\mathfrak{g}$-module $V$ such that the Chevalley involution $\Theta$ does not act as a scalar on $V[0]$. Thus, $V[0]$ is reducible under $\mathcal{C}_{\mathfrak{g}}$ and $\mathcal{C}_{\mathfrak{g}}$ and $Z(U \mathfrak{g})$ do not generate $U \mathfrak{g}^{\mathfrak{h}}$.

ProOF. By proposition 4.6, it suffices to exhibit an irreducible representation $V$ of $\mathfrak{g l}_{n}$ which is self-dual as $\mathfrak{s l}_{n}$-module and a Gelfand-Zetlin pattern $\mu$ describing a zero-weight vector for $\mathfrak{s l}_{n}$ in $V$ such that, for some $2 \leq k \leq n-1$, the $\mathfrak{s l}_{k}$-module $U$ with highest weight $\mu^{(k)}=\left(\mu_{1}^{(k)}, \ldots, \mu_{k}^{(k)}\right)$ is not self-dual. Since the highest weight of $U^{*}$ is $\left(-\mu_{k}^{(k)}, \ldots,-\mu_{1}^{(k)}\right)$, such a $U$ is self-dual iff the sum $\mu_{i}^{(k)}+\mu_{k+1-i}^{(k)}$ does not depend upon $i=1 \ldots k$. The following is a suitable Gelfand-Zetlin pattern $\mu$ 


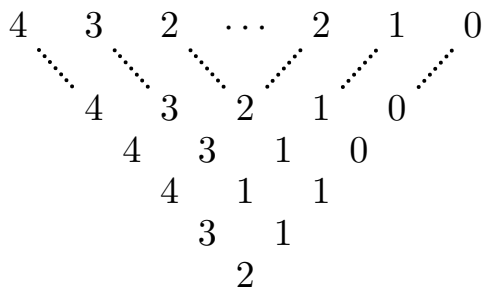

since the $\mathfrak{s l}_{3}$-module with highest weight $(4,1,1)$ is not self-dual and, for any $n \geq 4$, the $\mathfrak{s l}_{n}$-module with highest weight

$$
(4,3, \underbrace{2, \ldots, 2}_{n-4}, 1,0)
$$

is self-dual

\section{A conjecture of Kwon and Lusztig on quantum Weyl groups}

5.1. We discuss below some results of Kwon on $q$-Weyl group actions of Artin's braid group $B_{n}$ on the zero weight spaces of $U_{\hbar} \mathfrak{s l}_{n}$-modules [Kw]. We disprove in particular a conjecture of his and Lusztig's stating the irreducibility of all such representations.

Let $U_{\hbar} \mathfrak{g}$ be the Drinfeld-Jimbo quantum group corresponding to $\mathfrak{g}$ [Dr1, Ji], which we regard as a Hopf algebra over the ring $\mathbb{C} \llbracket \hbar \rrbracket$ of formal power series in the variable $\hbar$. By a finite-dimensional representation of $U_{\hbar \mathfrak{g}}$ we shall mean a $U_{\hbar \mathfrak{g}} \mathfrak{- m o d u l e} \mathcal{V}$ which is topologically free and finitely-generated over $\mathbb{C} \llbracket \hbar \rrbracket$. The isomorphism class of such a representation is uniquely determined by that of the $\mathfrak{g}$-module $V=\mathcal{V} / \hbar \mathcal{V}[\mathrm{Dr} 2]$.

Lusztig, and independently Kirillov-Reshetikhin and Soibelman $[\mathrm{Lu}, \mathrm{KR}$, So], proved that any such $\mathcal{V}$ carries an action, called the quantum Weyl group action of the braid group $B_{\mathfrak{g}}$. Its reduction mod $\hbar$ factors through the Tits extension $\widetilde{W}$ given by the triple exponentials (2.13) with $t_{i}=1$. Specifically, this action is given by mapping the generator $S_{i}$ of $B_{\mathfrak{g}}$ to the triple $q$-exponential [Sa]

$$
\begin{aligned}
& \exp _{q_{i}^{-1}}\left(q_{i}^{-1} E_{i} q_{i}^{-H_{i}}\right) \exp _{q_{i}^{-1}}\left(-F_{i}\right) \exp _{q_{i}^{-1}}\left(q_{i}^{-1} E_{i} q_{i}^{-H_{i}}\right) q_{i}^{H_{i}\left(H_{i}+2\right) / 2} \\
= & \exp _{q_{i}^{-1}}\left(-q_{i}^{-1} F_{i} q_{i}^{H_{i}}\right) \exp _{q_{i}^{-1}}\left(E_{i}\right) \exp _{q_{i}^{-1}}\left(-q_{i}^{-1} F_{i} q_{i}^{H_{i}}\right) q_{i}^{H_{i}\left(H_{i}+2\right) / 2}
\end{aligned}
$$

where $E_{i}, F_{i}, H_{i}$ are the generators of $U_{\hbar} \mathfrak{g}$ corresponding to the simple root $\alpha_{i}, q_{i}=q^{\left\langle\alpha_{i}, \alpha_{i}\right\rangle \hbar}$ and the $q$-exponential is defined by

$$
\exp _{q}(X)=\sum_{n \geq 0} \frac{q^{n(n-1) / 2}}{[n]_{q} !} X^{n}
$$


where the $q$-factorials are given by

$$
[n]_{q}=\frac{q^{n}-q^{-n}}{q-q^{-1}} \quad \text { and } \quad[n]_{q} !=[n]_{q}[n-1]_{q} \cdots[1]_{q}
$$

Kwon investigated the $q$-Weyl group action of Artin's braid group $B_{n}=$ $B_{\mathfrak{s} l_{n}}$ on the zero weight space of a simple $U_{\hbar} \mathfrak{s l}_{n}$-module $\mathcal{V}$. He gave a general criterion for it to be irreducible $[\mathrm{Kw}]^{5}$ and showed moreover that this criterion holds for all representations of $U_{\hbar \mathfrak{s l}_{3}}$. From these findings, he and Lusztig conjectured that the action of $B_{n}$ on $\mathcal{V}[0]$ is irreducible for any simple $U_{\hbar} \mathfrak{s l}_{n}$-module $\mathcal{V}$. We shall prove in this section the following

Theorem 5.1. The Kwon-Lusztig conjecture is false for any complex, simple Lie algebra $\mathfrak{g}$ not isomorphic to $\mathfrak{s l}_{2}, \mathfrak{s l}_{3}$.

5.2. Classical and Quantum Chevalley involution. Let $\Theta_{\hbar}$ be the quantum Chevalley involution, i.e., the algebra automorphism of $U_{\hbar} \mathfrak{g}$ defined by

$$
\Theta_{\hbar}\left(E_{i}\right)=-F_{i}, \quad \Theta_{\hbar}\left(F_{i}\right)=-E_{i} \quad \text { and } \quad \Theta_{\hbar}\left(H_{i}\right)=-H_{i}
$$

As in the classical case, $\Theta_{\hbar}$ acts on any self-dual finite-dimensional representation of $U_{\hbar} \mathfrak{g}$ leaving its zero weight space invariant. Since $H_{i}$ acts as zero on $\mathcal{V}[0]$, we see from (5.1) that $\Theta_{\hbar}$ centralises $B_{\mathfrak{g}}$ on $\mathcal{V}[0]$. Corollary 5.3 below relates the action of $\Theta_{\hbar}$ on $\mathcal{V}[0]$ to that of the classical Chevalley involution $\Theta$ on $V$ [0]. It will be used to deduce theorem 5.1 from the results of section 4 .

Proposition 5.2. There exists an algebra isomorphism $\Psi: U_{\hbar} \mathfrak{g} \rightarrow U \mathfrak{g} \llbracket \hbar \rrbracket$ which is equal to the identity mod $\hbar$, acts as the identity on $\mathfrak{h}$ and satisfies

$$
\Psi \circ \Theta_{\hbar} \circ \Psi^{-1}=\Theta
$$

Proof. Let $\Phi: U_{\hbar \mathfrak{g}} \rightarrow U \mathfrak{g} \llbracket \hbar \rrbracket$ be an algebra isomorphism equal to the identity $\bmod \hbar$ and acting as the identity on $\mathfrak{h}$ [Dr2, Prop. 4.3]. Set

$$
\widetilde{\Theta}=\Phi \circ \Theta_{\hbar} \circ \Phi^{-1}=\Theta \circ \delta
$$

where $\delta \in \mathrm{id}+\hbar \cdot \operatorname{End}(U \mathfrak{g}) \llbracket \hbar \rrbracket$ is such that $\delta(p)=p$ for any $p \in U \mathfrak{h}$. The involutivity of $\widetilde{\Theta}$ yields $\Theta \circ \delta=\delta^{-1} \circ \Theta$ so that $a=\delta^{1 / 2}$ satisfies $a \circ \widetilde{\Theta}=\Theta \circ a$ and $\Psi=a \circ \Phi$ is the required isomorphism

Corollary 5.3. Let $\mathcal{V}$ be a self-dual, finite-dimensional $U_{\hbar} \mathfrak{g}-$ module and let $V=\mathcal{V} / \hbar \mathcal{V}$ be its reduction $\bmod \hbar$. Then, $\mathcal{V}$ and $V \llbracket \hbar \rrbracket$ are isomorphic as $\mathfrak{h} \rtimes \mathbb{Z}_{2}$-modules where the generator of $\mathbb{Z}_{2}$ acts as $\Theta_{\hbar}$ on $\mathcal{V}$ and as the classical Chevalley involution $\Theta$ on $V$. In particular, $\Theta_{\hbar}$ acts as a scalar on $\mathcal{V}[0]$ iff it acts as a scalar on $V[0]$.

\footnotetext{
5 when coefficients are extended to the field $\mathbb{C}((\hbar))$ of formal Laurent series, which we tacitly assume.
} 
Proof. The isomorphism $\Psi: U \mathfrak{g} \llbracket \hbar \rrbracket \rightarrow U \mathfrak{g} \llbracket \hbar \rrbracket$ of proposition 5.2 endows $V \llbracket \hbar \rrbracket$ with the structure of a $U_{\hbar} \mathfrak{g}$-module such that the action of $\mathfrak{h} \subset U_{\hbar} \mathfrak{g}$ coincides with that of $\mathfrak{h} \subset \mathfrak{g}$. Since $\mathcal{V}$ and $V \llbracket \hbar \rrbracket$ have the same reduction $\bmod \hbar$, they are isomorphic as $U_{\hbar} \mathfrak{g}$, and therefore $\mathfrak{h}$-modules. Equation (5.2) then guarantees that, under this isomorphism, the Chevalley involution of $\mathcal{V}$ is mapped to the Chevalley involution of $V$

Proof of THEOREM 5.1. By proposition 4.7 and theorem 4.10, there exists a simple, self-dual $\mathfrak{g}$-module $V$ such that $\Theta$ does not act as a scalar on $V[0]$. By corollary $5.3, \Theta_{\hbar}$ does not act as scalar on $V \llbracket \hbar \rrbracket[0]$ and the latter is reducible under the $q$-Weyl group action of $B_{\mathfrak{g}}$

We mention in passing the following $q$-analogue of proposition 3.11

Proposition 5.4. Let $\mathcal{V}$ be a finite-dimensional representation of $U_{\hbar} \mathfrak{g}$ with non-trivial zero weight space $\mathcal{V}[0]$. Then, $\mathcal{V}[0]$ is irreducible under $B_{\mathfrak{g}}$ iff it is irreducible under $P_{\mathfrak{g}}$.

Proof. By proposition 1.2.1 of [Sa], $S_{i}$ acts on the zero-weight space of the indecomposable $U_{\hbar} \mathfrak{s l}_{2}^{\alpha_{i}}$-module of dimension $2 n+1$ as multiplication by $(-1)^{n} q_{i}^{n(n+1)}$. It follows that the image of $S_{i}$ in $\operatorname{End}(\mathcal{V}[0])$ is a polynomial in the image of $S_{i}^{2} \in P_{\mathfrak{g}}$ whence the conclusion

5.3. A Kohno-Drinfeld theorem for $q-$ Weyl groups. Let $\mathcal{V}$ be a finitedimensional representation of $U_{\hbar} \mathfrak{g}$ and $V=\mathcal{V} / \hbar \mathcal{V}$ its reduction $\bmod \hbar$. It was conjectured in [TL2], by analogy with the Kohno-Drinfeld theorem, that the $q$-Weyl group action of $B_{\mathfrak{g}}$ on $\mathcal{V}$ is equivalent to to the monodromy action of $B_{\mathfrak{g}}$ on $\nabla_{\kappa}$ defined in the present paper ${ }^{6}$. This conjecture is proved in [TL3] for a number of pairs $(\mathfrak{g}, V)$ including vector representations of classical Lie algebras and adjoint representations of all simple Lie algebras and in [TL2] for all representations of $\mathfrak{g}=\mathfrak{s l}_{n}$. More precisely,

Theorem 5.5 ([TL2]). Assume that $\mathfrak{g} \cong \mathfrak{s l}_{n}$. Let $\mu$ be a weight of $V$ and

$$
V^{\mu}=\bigoplus_{\nu \in W \mu} V[\nu]
$$

the direct sum of the weight spaces of $V$ corresponding to the Weyl group orbit of $\mu$. Let $\sigma\left(B_{\mathfrak{g}}\right) \subset N(T)$ be a Tits extension and

$$
\rho^{\sigma}: B_{\mathfrak{g}} \rightarrow G L\left(V^{\mu} \llbracket h \rrbracket\right)
$$

the corresponding monodromy representation defined by proposition 2.3 by regarding $h$ as a formal variable. Let $\pi_{W}: B_{\mathfrak{g}} \rightarrow G L\left(\mathcal{V}^{\mu}\right)$ be the $q-W e y l$ group action. Then, $\rho_{h}$ and $\pi_{W}$ are equivalent for $\hbar=2 \pi i h$.

Combining the above theorem with corollary 3.12, we obtain the following

\footnotetext{
6 this conjecture was independently formulated by De Concini around 1995 (unpublished).
} 
Proposition 5.6. Let $\mathcal{V}$ be a finite-dimensional representation of $U_{\hbar} \mathfrak{s l}_{n}$ and set $V=\mathcal{V} / \hbar \mathcal{V}$. The following statements are equivalent

(i) $\mathcal{V}[0]$ is (absolutely) irreducible under the $q$-Weyl group action of $B_{n}$.

(ii) $\mathcal{V}[0]$ is (absolutely) irreducible under the $q$-Weyl group action of $P_{n}$.

(iii) $V[0]$ is irreducible under the Casimir algebra $\mathcal{C}_{\mathfrak{s l}_{n}}$.

In particular, by theorem 4.1, the $q$-Weyl group action of $P_{3}$ on the zero weight space $\mathcal{V}[0]$ of a $U_{\hbar} \mathfrak{s l}_{3}$-module is always irreducible, a slight refinement of a result of Kwon asserting the irreducibility of $\mathcal{V}[0]$ under the full braid group $B_{3}$.

\section{IrReducible RePRESEntations of $\mathcal{C}_{\mathfrak{g}}$}

The aim of this section is to show that the connection $\nabla_{\kappa}$ yields irreducible monodromy representations of $B_{\mathfrak{g}}$ of arbitrarily large dimension. For $\mathfrak{g}=\mathfrak{s l}_{n}$, we show for example $\S 6.1$ that the weight spaces of all Cartan powers of the adjoint representation are irreducible under the Casimir algebra $\mathcal{C}_{\mathfrak{g}}$. For $\mathfrak{g} ¥ \mathfrak{s l}_{n}$, we obtain in $\S 6.2$ a somewhat weaker result : for every $p \in \mathbb{N}$, the zero weight space of the $p$ th Cartan power of $\operatorname{ad}(\mathfrak{g})$ has a subspace $K_{p}$ which is irreducible under $\mathcal{C}_{\mathfrak{g}}$ and such that $\lim _{p \rightarrow+\infty} \operatorname{dim} K_{p}=+\infty$.

\subsection{Irreducible representations of $\mathcal{C}_{\mathfrak{s l}_{n}}$.}

Theorem 6.1. For any $p, q \in \mathbb{N}$, the action of $\mathcal{C}_{\mathfrak{s l}_{n}}$ on the weight spaces of the simple $\mathfrak{s l}_{n}$-module of highest weight $(p, 0, \ldots, 0,-q)$ is irreducible.

Proof. For any $2 \leq k \leq n$ and $a, b \in \mathbb{N}$, set $\lambda_{a, b}^{(k)}=(a, 0, \ldots, 0,-b) \in \mathbb{Z}^{k}$ so that the Gelfand-Zetlin basis of the irreducible $\mathfrak{g l}_{n}$-module $V$ with highest weight $\lambda_{p, q}^{(n)}$ is parametrised by patterns of the form

$$
\lambda=\left(\begin{array}{c}
\lambda_{p_{n}, q_{n}}^{(n)} \\
\vdots \\
\lambda_{p_{k}, q_{k}}^{(k)} \\
\vdots \\
\lambda_{p_{2}, q_{2}}^{(2)} \\
r
\end{array}\right)
$$

where the $p_{k}=p_{k}(\lambda), q_{k}=q_{k}(\lambda)$ and $r=r(\lambda)$ are integers satisfying

$$
\begin{gathered}
p=p_{n} \geq p_{n-1} \geq \cdots \geq p_{2} \geq 0 \\
q=q_{n} \geq q_{n-1} \geq \cdots \geq q_{2} \geq 0 \\
p_{2} \geq r \geq-q_{2}
\end{gathered}
$$

The vectors of a given weight $\mu=\left(\mu_{1}, \ldots, \mu_{n}\right)$ correspond to patterns satisfying in addition

$$
r=\mu_{1} \quad \text { and, for any } 2 \leq k \leq n, \quad p_{k}-q_{k}=M_{k}
$$


where $M_{k}=\sum_{i=1}^{k} \mu_{k}$. We claim that the commuting Casimir operators $C_{\mathfrak{g l}_{k}}, 2 \leq k \leq n$, have joint simple spectrum on the weight space $V[\mu]$, with corresponding diagonal basis given by Gelfand-Zetlin vectors. Indeed, $C_{\mathfrak{g l}_{k}}$ acts on $v_{\lambda} \in V[\mu]$, with $\lambda$ of the form (6.1), as multiplication by

$$
\begin{aligned}
\left\langle\lambda_{p_{k}, q_{k}}^{(k)}, \lambda_{p_{k}, q_{k}}^{(k)}+2 \rho^{(k)}\right\rangle & =p_{k}^{2}+q_{k}^{2}+(k-1)\left(p_{k}+q_{k}\right) \\
& =2 q_{k}^{2}+2 q_{k}\left(M_{k}+k-1\right)+M_{k}\left(M_{k}+k-1\right)
\end{aligned}
$$

where $2 \rho^{(k)}=\sum_{i=1}^{k} \theta_{i}(k-2 i+1)$ is the sum of the positive roots of $\mathfrak{g l}_{k}$. Since $2 q_{k}+M_{k}=q_{k}+p_{k} \geq 0$ and the the right-hand side of (6.2) is a parabola with vertex at

$$
q_{k}^{0}=-\frac{1}{2}\left(M_{k}+k-1\right)<-\frac{M_{k}}{2}
$$

the $C_{\mathfrak{g l}_{k}}$-eigenvalue of a pattern $\lambda$ of form (6.1) and weight $\mu$ determines $p_{k}(\lambda)$ and $q_{k}(\lambda)$ uniquely as claimed.

We claim now that if $K \subseteq V[\mu]$ is a non-zero subspace invariant under $\mathcal{C}_{\mathfrak{s l}_{n}}$, then $K=V[\mu]$. To see this, it suffices to show that, for any given pattern $\lambda$ of the form (6.1) and weight $\mu$, there exists a Gelfand-Zetlin vector lying in $K$ such that the corresponding pattern has the same $(n-1)$ row $\lambda_{p_{n-1}, q_{n-1}}^{(n-1)}$ as $\lambda$, for then a descending induction on $n$ shows that $K$ contains all GelfandZetlin vectors of weight $\mu$. Since the Casimirs $C_{\mathfrak{g l}_{k}}$ have simple spectrum on $V[\mu], K$ contains at least one Gelfand-Zetlin vector $v_{\lambda^{\prime}}$. Let $\lambda_{p_{n-1}^{\prime}, q_{n-1}^{\prime}}^{(n-1)}$ be the $n-1$ row of the corresponding pattern. If $p_{n-1}^{\prime}=p_{n-1}$, then,

$$
q_{n-1}^{\prime}=-M_{n-1}+p_{n-1}^{\prime}=-M_{n-1}+p_{n-1}=q_{n-1}
$$

and we are done. If $p_{n-1}^{\prime}<p_{n-1}(\lambda)$, we may further assume that $p_{n-1}^{\prime}=$ $\max _{\widetilde{\lambda}} p_{n-1}(\widetilde{\lambda})$, where the maximum is taken over all patterns $\widetilde{\lambda}$ such that $v_{\tilde{\lambda}} \in K$ and $p_{n-1}(\widetilde{\lambda}) \leq p_{n-1}$. Note then that

$$
q_{n-1}^{\prime}=-\sum_{i=1}^{n-1} \mu_{i}+p_{n-1}^{\prime}<-\sum_{i=1}^{n-1} \mu_{i}+p_{n-1}=q_{n-1} \leq q_{n}
$$

It therefore follows by corollary 4.9 that

$$
\begin{aligned}
\kappa_{\theta_{n-1}-\theta_{n}} v_{\lambda^{\prime}} & =\left(2 E_{n-1, n} E_{n, n-1}+\left(E_{n-1, n-1}-E_{n, n}\right)\right) v_{\lambda^{\prime}} \\
& =a v_{\lambda^{\prime}+\varepsilon_{1}^{(n-1)}-\varepsilon_{n-1}^{(n-1)}}+b v_{\lambda^{\prime}-\varepsilon_{1}^{(n-1)}+\varepsilon_{n-1}^{(n-1)}}+c v_{\lambda^{\prime}}
\end{aligned}
$$

for some $a, b, c \in \mathbb{C}$ with $a \neq 0$. Hence, $K$ contains $v_{\lambda^{\prime}+\varepsilon_{1}^{(n-1)}-\varepsilon_{n-1}^{(n-1)}}$ in contradiction with the maximality of $p_{n-1}^{\prime}$. The case $p_{n-1}^{\prime}>p_{n-1}$ follows similarly

REMARK. A similar proof shows that the Casimir algebra $C_{\mathfrak{s l}_{n}}$ acts irreducibily on all weight spaces of the irreducible representations with highest weight of the form $(p, q, 0, \ldots, 0)$ where $p, q \in \mathbb{Z}$ satisfy $p \geq q \geq 0$. More 
generally, one can show that if the commuting Casimirs $C_{\mathfrak{g l}_{k}}, k=2 \ldots n$, have joint simple spectrum on the weight space $V[\mu]$ of a simple $\mathfrak{s l}_{n}$-module $V$, then $\mathcal{C}_{\mathfrak{s l}}$ acts irreducibly on $V[\mu]$. The proof is similar to that of theorem 6.1 but somewhat more involved technically and will be given in a future publication.

6.2. Irreducible representations of $\mathcal{C}_{\mathfrak{g}}, \mathfrak{g} \not \mathfrak{s l}_{n}$. Let $\mathfrak{g}$ be a complex, simple Lie algebra not isomorphic to $\mathfrak{s l}_{n}$ and let $\theta$ be the highest root of $\mathfrak{g}$.

Theorem 6.2. For any $p \in \mathbb{N}$, there exists a subspace $K_{p}$ of the zero weight space of the simple $\mathfrak{g}$-module with highest weight $p \theta$ which is irreducible under $\mathcal{C}_{\mathfrak{g}}$ and such that $\lim _{p \rightarrow \infty} \operatorname{dim} K_{p}=\infty$.

Proof. We shall need the following simple

Lemma 6.3. Let $R$ be a root system and $\alpha \neq \pm \beta \in R$ two long roots which are not orthogonal. Then, $R \cap(\mathbb{Z} \alpha+\mathbb{Z} \beta)$ is a root system of type $A_{2}$.

Proof. Since $\|\alpha\|=\|\beta\|$, one has $\left\langle\alpha, \beta^{\vee}\right\rangle= \pm 1$. Replacing $\beta$ by $-\beta$ if necessary, we may assume that $\left\langle\alpha, \beta^{\vee}\right\rangle=-1$. Thus, $\pm \alpha, \pm \beta, \pm(\alpha+\beta)=$ $\pm \sigma_{\beta} \alpha \in R$ and these are the only $\mathbb{Z}$-linear combinations of $\alpha, \beta$ which lie in $R$ since any other has norm strictly larger than $\|\alpha\|$

Assume first that $\mathfrak{g} ¥ \mathfrak{s p}_{2 n}, n \geq 2$. An inspection of the tables in [Bo] then shows that there is a unique simple root $\alpha$ of $\mathfrak{g}$ which is not orthogonal to $\theta$ and is, moreover, long. Applying the above lemma to the pair $(\alpha, \theta)$ yields a subalgebra

$$
\mathfrak{l}=\mathbb{C} h_{\alpha} \oplus \mathbb{C} h_{\theta} \bigoplus_{\gamma \in R(\mathfrak{g}) \cap(\mathbb{Z} \alpha+\mathbb{Z} \theta), \gamma \succ 0} \mathbb{C} e_{\gamma} \oplus \mathbb{C} f_{\gamma} \subset \mathfrak{g}
$$

which is isomorphic to $\mathfrak{s l}_{3}$ and has as highest root vector $e_{\theta}$. Choose $\mathfrak{h}_{\mathfrak{l}}=$ $\mathbb{C} h_{\alpha} \oplus \mathbb{C} h_{\theta}$ as Cartan subalgebra of $\mathfrak{l}$ and denote by $\mathfrak{l}^{* p}$ (resp. $\mathfrak{g}^{* p}$ ) the irreducible representation of $\mathfrak{l}$ (resp. $\mathfrak{g}$ ) with highest weight $p \theta$. Since $\mathfrak{l}^{* p}$ is generated by $e_{\theta}^{\otimes p}$ inside $\mathfrak{l}^{\otimes p} \subset \mathfrak{g}^{\otimes p}$, it follows that $\mathfrak{g}^{* p}$ contains $\mathfrak{l}^{* p}$ as $\mathfrak{l}$-submodule. This inclusion induces one of weight spaces $\mathfrak{l}^{* p}[0] \subset \mathfrak{g}^{* p}[0]$ since $\mathfrak{h}=\mathfrak{h}_{\mathfrak{l}} \oplus \mathfrak{h}_{\mathfrak{l}}^{\perp}$ and $\mathfrak{h}_{\mathfrak{l}}^{\perp}$ centralises $\mathfrak{l}$. By theorem 4.1, $\mathfrak{l}^{* p}[0]$ is irreducible under $\mathcal{C}_{\mathfrak{l}}$. Let $U=\mathcal{C}_{\mathfrak{g}}{ }^{* p}[0]$ be the $\mathcal{C}_{\mathfrak{g}}$-submodule of $\mathfrak{g}^{* p}[0]$ generated by ${ }^{*}{ }^{* p}[0]$ and decompose it as a sum $\bigoplus_{i} U_{i}$ of irreducible summands with projections $p_{i}$. By Schur's lemma, the restriction of each $p_{i}$ to $\mathfrak{l}^{* p}[0]$ is either zero or injective. Thus, the dimension of at least one of the $U_{i}$ 's is greater or equal to that of $\mathfrak{l}^{* p}[0]$ and therefore tends to infinity with $p$. If $\mathfrak{g} \cong \mathfrak{s p}_{2 n}$, the Koike-Terada branching rules [KT, thm. A1] show that $\operatorname{res}_{\mathfrak{g}}^{\mathfrak{s h}_{h}} \mathfrak{g}^{* p} \supset \mathfrak{s l}_{n}{ }^{* p}$ and we may conclude as above with $l=\mathfrak{s l}_{n}$

\section{ZERO WEIGHT SPACES OF SELF-DUAL $\mathfrak{g}$-MODULES}

The aim of this section is to show that, when $\mathfrak{g}$ is isomorphic to $\mathfrak{s l}_{n}, n \geq 4$, or $\mathfrak{g}_{2}$, the zero weight space of most self-dual, simple $\mathfrak{g}$-modules is reducible 
under the Casimir algebra $\mathcal{C}_{\mathfrak{g}}$, thus strengthening the results of section 4 . We classify the self-dual $V$ with $V[0]$ irreducible under $\mathcal{C}_{\mathfrak{s l}_{n}}$ and $\mathcal{C}_{\mathfrak{g}_{2}}$ in 7.1 and 7.2 respectively. Our calculations rely on the use of the Chevalley involution $\Theta$ via proposition 4.6. They show in fact that $V[0]$ is irreducible under $\mathcal{C}_{\mathfrak{g}}$ if, and only if $\Theta$ acts as a scalar on it. We conjecture in 7.3 that this holds for any simple Lie algebra $\mathfrak{g}$.

REMARK. By corollary 5.3, our results imply that the $q$-Weyl group action of $B_{\mathfrak{g}}$ on the zero weight spaces of most self-dual $U_{\hbar} \mathfrak{g}$-modules is reducible.

\subsection{Self-dual representations of $\mathfrak{s l}_{n}$ •}

Theorem 7.1. Let $V$ be a simple, self-dual $\mathfrak{s l}_{n}$-module with non-trivial zero weight space $V[0]$. Then, $V[0]$ is irreducible under the Casimir algebra $\mathcal{C}_{\mathfrak{s l}_{n}}$ if the highest weight $\lambda$ of $V$ is of one of the following forms

(i) $\lambda=(p, 0, \ldots, 0,-p), p \in \mathbb{N}$.

(ii) $\lambda=(\underbrace{1, \ldots, 1}_{k}, 0, \ldots, 0, \underbrace{-1, \ldots,-1}_{k}), 0 \leq k \leq n / 2$

(iii) $\lambda=(p, p,-p,-p), p \in \mathbb{N}$.

Conversely, if $\lambda$ is of none of the above forms, there exists $a k<n$ and $a$ simple $\mathfrak{g l}_{k}$-summand $U \subset V$ with $U \not U^{*}$ and $U \cap V[0] \neq\{0\}$. In particular, $V[0]$ is reducible under $\mathcal{C}_{\mathfrak{s l}_{n}}$.

Since the case (i) follows from theorem 6.1, the "if" part of theorem 7.1 is settled by the following two lemmas.

Lemma 7.2. Let $V_{n, k}$ be the simple $\mathfrak{s l}_{n}$-module with highest weight

$$
\lambda_{k}^{(n)}=(\underbrace{1, \ldots, 1}_{k}, 0, \ldots, 0, \underbrace{-1, \ldots,-1}_{k})
$$

Then $V_{n, k}[0]$ is irreducible under $\mathcal{C}_{\mathfrak{s l}_{n}}$.

Proof. We claim that the Casimirs $C_{\mathfrak{g l}_{m}}, m=2, \ldots n$, have joint simple spectrum on $V_{n, k}[0]$. Indeed, the $m$ th row of a zero weight Gelfand-Zetlin pattern corresponding to $V_{n, k}$ is of the form $\lambda_{l}^{(m)}$, for some $0 \leq l \leq m / 2$. Since $C_{\mathfrak{g l}_{m}}$ acts as multiplication by $2 l(m-l+1)$ on the representation with highest weight $\lambda_{l}^{(m)}$ and the function $f(x)=2 x(m-x+1)$ is injective on the interval $[0,(m+1) / 2]$, the $C_{\mathfrak{g l}_{m}}$-eigenvalue of a zero weight GelfandZetlin pattern determines its $m$ th row uniquely, as claimed. The proof is now completed as in theorem 6.1

Lemma 7.3. For any $p \in \mathbb{N}$, let $V_{p}$ be the simple $\mathfrak{s l}_{4}$-module with highest weight $(p, p,-p,-p)$. Then, $V_{p}[0]$ is irreducible under $\mathcal{C}_{\mathfrak{s l}_{4}}$. 
Proof. The Gelfand-Zetlin patterns corresponding to $V_{p}[0]$ are of the form

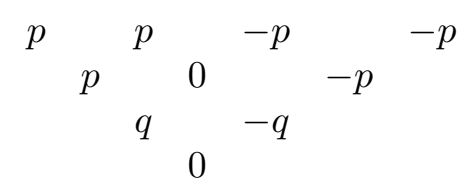

for some $0 \leq q \leq p$, so that they are separated by the action of the Casimir of $\mathfrak{s l}_{2} \subset \mathfrak{s l}_{n}$. The proof is now completed as in theorem 6.1

Proof of Theorem 7.1. Assume that $\lambda$ is not of the form (i)-(iii) and let

$$
s(\lambda)=\left|\left\{i=1 \ldots n-1 \mid \lambda_{i}-\lambda_{i+1}>0\right\}\right|
$$

be the number of steps in the corresponding Young diagram. Noting that $s(\lambda)=2$ for the highest weights of the form (i)-(iii), we begin by proving that $V[0]$ is reducible under $\mathcal{C}_{\mathfrak{s l}_{n}}$ if $s(\lambda) \geq 3$. Suppose first that $n=2 k+1$ is odd. Since $V[0] \neq\{0\}$, we may assume that the sum $|\lambda|$ of the entries in $\lambda$ is zero so that, by self-duality, $\lambda$ is of the form

$$
\lambda=\left(a_{1}, \ldots, a_{l}, 0, \ldots, 0,-a_{l}, \ldots,-a_{1}\right)
$$

for some $a_{1} \geq \cdots \geq a_{l}>0$, with at least one middle zero. Since $s(\lambda) \geq 3$, there exists some $1 \leq i \leq l-1$ such that $a_{i}>a_{i+1}$. Let $\mu$ be the $\mathfrak{s l}_{n-1^{-}}$ weight obtained by replacing $a_{i}$ by $a_{i}-1$ in the $i$ th position, $-a_{l}$ by $-a_{l}+1$ in the $n-l+1$ th position and by omitting the middle zero. Then, $|\mu|=$ $\sum_{i=1}^{n-1} \mu_{i}=|\lambda|=0$ and

$$
\mu_{i}+\mu_{n-i}=-1 \neq 1=\mu_{l}+\mu_{n-l}
$$

so that $\mu$ is a non-self dual weight of $\mathfrak{s l}_{n-1}$ such that $V_{\mu} \cap V[0] \neq\{0\}$.

Consider now the case where $n=2 k$ is even. Assuming again that $|\lambda|=0$, we find that

$$
\lambda=(a_{1}, \ldots, a_{l}, \underbrace{0, \ldots, 0}_{n_{0}},-a_{l}, \ldots,-a_{1})
$$

where the number $n_{0}$ of zeroes is even. If $n_{0}>0$, the $\mathfrak{s l}_{n-1}$ weight $\mu$ obtained by replacing the two middle zeroes by a single one in $\lambda$ is selfdual and satisfies $|\mu|=0$ and $s(\mu)=s(\lambda)$. By our previous analysis, there therefore exists a non-self dual $\mathfrak{s l}_{n-2}$ weight $\nu \prec \mu \prec \lambda$ such that $|\nu|=0$ and $V_{\nu} \cap V[0] \neq 0$. If, on the other hand, $n_{0}=0$, then

$$
\lambda=\left(a_{1}, \cdots, a_{n / 2},-a_{n / 2}, \cdots,-a_{1}\right)
$$

with $a_{n / 2}>0$. Let $1 \leq i \leq n / 2-1$ be such that $a_{i}>a_{i+1}$. Then, the non-self dual $\mathfrak{s l}_{n-1}$-weight $\mu \prec \lambda$ obtained by replacing $a_{i}$ by $a_{i}-1$ in the $i$ th position and the pair $a_{n / 2},-a_{n / 2}$ allows to conclude. 
Consider now the case $s(\lambda) \leq 2$. By self-duality, we may take $\lambda$ of the form

$$
\lambda=(\underbrace{p, \ldots, p}_{k}, 0, \ldots, 0, \underbrace{-p, \ldots,-p}_{k})
$$

for some $0 \leq k \leq n / 2$. By assumption, $k \geq 2$ and $p>1$ since $\lambda$ is not of the forms (i)-(ii). If $n$ is odd, replacing the innermost pair $(p,-p)$ by $(p-1,-(p-1))$ and suppressing the middle zero yields an $\mathfrak{s l}_{n-1}$ weight $\mu$ with $s(\mu) \geq 3$ and our previous analysis allows to conclude. If $n$ is even and there are two or more middle zeroes, we suppress one of them to obtain an $\mathfrak{s l}_{n-1}$-weight $\nu$ of of the form treated in the previous paragraph. If there are no middle zeroes, then by assumption $n \geq 6$. We change the innermost pair $(p,-p)$ to 0 and proceed as above

7.2. Representations of $\mathfrak{g}_{2}$. Recall that, for $\mathfrak{g}=\mathfrak{g}_{2}$, every $\mathfrak{g}$-module $V$ is self-dual and has a non-trival zero weight space. The aim of this subsection is to prove the following.

Theorem 7.4. The zero weight space $V[0]$ of a simple $\mathfrak{g}_{2}$-module $V$ is irreducible under the Casimir algebra $\mathcal{C}_{\mathfrak{g}_{2}}$ iff $V$ is a trivial or fundamental representation, or its second Cartan power.

The proof of the theorem is given in the next three propositions. We begin by reviewing Perroud's branching rules for the equal rank inclusion $\mathfrak{s l}_{3} \subset \mathfrak{g}_{2}$ [Pe]. Let $\alpha_{1}, \alpha_{2}$ be the long and short simple roots of $\mathfrak{g}_{2}$ respectively and $\varpi_{1}, \varpi_{2}$ the corresponding fundamental weights ${ }^{7}$. Let $V_{\lambda}$ be the simple $\mathfrak{g}_{2}-$ module with highest weight $\lambda=m_{1} \varpi_{1}+m_{2} \varpi_{2}$. Consider the set of GelfandZetlin patterns $\mu(a, b, c)$ of the form

$$
\begin{array}{cccc}
m_{1}+m_{2} & & m_{2} & \\
& a & & \\
& & & \\
& & &
\end{array}
$$

Then,

$$
\operatorname{res}_{\mathfrak{g}_{2}}^{\mathfrak{s l}_{3}} V_{\lambda}=\bigoplus_{\mu(a, b, c)} V_{\left(m_{1}+c, a-m_{2}+b, 0\right)}
$$

Lemma 7.5. For any complex, simple Lie algebra $\mathfrak{g}, \mathfrak{h}=\operatorname{ad}(\mathfrak{g})[0]$ is irreducible under $\mathcal{C}_{\mathfrak{g}}$.

Proof. This follows from proposition 3.11 since $\mathfrak{h}$ is irreducible under $W$

Proposition 7.6. The zero weight spaces of the fundamental representations of $\mathfrak{g}$ and of their second Cartan powers are irreducible under $\mathcal{C}_{\mathfrak{g}}$.

Proof. The zero weight space of $V_{\varpi_{2}} \cong \mathbb{C}^{7}$ is one-dimensional and therefore irreducible under $\mathcal{C}_{\mathfrak{g}}$. The branching rules (7.2) yield

$$
\operatorname{res}_{\mathfrak{g}_{2}}^{\mathfrak{s l}_{3}} V_{2 \varpi_{2}}=\operatorname{ad}\left(\mathfrak{s l}_{3}\right) \oplus R
$$

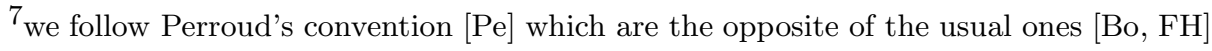


where $R$ is a reducible $\mathfrak{s l}_{3}$-modules with trivial zero weight space. The irreducibility of $V_{2 \varpi_{2}}[0]$ therefore follows from lemma 7.5 for $\mathfrak{g}=\mathfrak{s l}_{3}$ or from theorem 4.1. Since $V_{\varpi_{1}} \cong \operatorname{ad}\left(\mathfrak{g}_{2}\right)$, the irreducibility of $V_{\varpi_{1}}[0]$ under $\mathcal{C}_{\mathfrak{g}}$ follows from lemma 7.5. Finally, by (7.2),

$$
\operatorname{res}_{\mathfrak{g}}^{\mathfrak{s l}_{3}} C^{2} \operatorname{ad}(\mathfrak{g})=C^{2} \operatorname{ad}\left(\mathfrak{s l}_{3}\right) \oplus V \oplus R
$$

where

$$
\begin{aligned}
& V=V_{(2,1,0)} \cong \operatorname{ad}\left(\mathfrak{s l}_{3}\right) \\
& R=V_{(3,2,0)} \oplus V_{(3,1,0)} \oplus V_{(2,2,0)} \oplus V_{(2,0,0)}
\end{aligned}
$$

and $R$ has a trivial 0 weight space. One readily checks that $C^{2} \operatorname{ad}\left(\mathfrak{s l}_{3}\right)$ and $V$ are distinguished by the Casimir eigenvalue of $\mathfrak{s l}_{3}$ so that, by theorem 4.1, the zero weight spaces of $C^{2} \operatorname{ad}\left(\mathfrak{s l}_{3}\right)$ and $V$ are irreducible and inequivalent representations of $\mathcal{C}_{\mathfrak{s l}_{3}}$. Since $C^{2} \operatorname{ad}(\mathfrak{g})[0]=C^{2} \operatorname{ad}\left(\mathfrak{s l}_{3}\right)[0] \oplus V[0]$ it suffices to show that $C^{2} \operatorname{ad}\left(\mathfrak{s l}_{3}\right)[0]$ is not invariant under $\mathcal{C}_{\mathfrak{g}}$. Let $\theta$ be the highest root of $\mathfrak{g}$ and $e_{\theta}, f_{\theta}, h_{\theta}$ a corresponding $\mathfrak{s l}_{2}^{\theta}$ triple. This triple lies in $\mathfrak{s l}_{3}$ so that

$$
v_{\theta}=-1 / 4 \operatorname{ad}\left(f_{\theta}\right) \operatorname{ad}\left(e_{\theta}\right) e_{\theta}^{2}=e_{\theta} \cdot f_{\theta}-\frac{1}{2} h_{\theta}^{2} \in C^{2} \operatorname{ad}\left(\mathfrak{s l}_{3}\right) \subset C^{2} \operatorname{ad}(\mathfrak{g})
$$

where we are realising $C^{2} \operatorname{ad}(\mathfrak{g})$ as the highest weight component of $S^{2} \mathfrak{g}$. Let $\alpha$ be a short root of $\mathfrak{g}$ such that $\left\langle\theta^{\vee}, \alpha\right\rangle=1$ so that the $\alpha$-string through $\theta$ is of the form $\theta-2 \alpha, \theta-\alpha, \theta$. It is easy to see that such an $\alpha$ exists by consulting the tables in $[\mathrm{Bo}]$. A simple computation using a Chevalley basis of $\mathfrak{g}$ yields

$$
\begin{aligned}
-\frac{1}{2} \operatorname{ad}\left(f_{\alpha}\right) \operatorname{ad}\left(e_{\alpha}\right) h_{\theta}^{2} & =e_{\alpha} \cdot f_{\alpha}-h_{\alpha} \cdot h_{\theta} \\
\operatorname{ad}\left(f_{\alpha}\right) \operatorname{ad}\left(e_{\alpha}\right) e_{\theta} \cdot f_{\theta} & = \pm e_{\theta-\alpha} \cdot f_{\theta-\alpha} \pm 2 e_{\theta} \cdot f_{\theta}
\end{aligned}
$$

where the signs depend on the choice of the root vectors. Thus,

$$
\frac{1}{\langle\alpha, \alpha\rangle} C_{\alpha} v_{\theta}=\operatorname{ad}\left(f_{\alpha}\right) \operatorname{ad}\left(e_{\alpha}\right) v_{\theta}=e_{\alpha} \cdot f_{\alpha} \pm e_{\theta-\alpha} \cdot f_{\theta-\alpha} \pm 2 e_{\theta} \cdot f_{\theta}-h_{\alpha} \cdot h_{\theta}
$$

which does not lie in $C^{2} \operatorname{ad}\left(\mathfrak{s l}_{3}\right)$ since $\alpha$ is short

Proposition 7.7. Let $V$ be a simple $\mathfrak{g}_{2}$-module with highest weight $\lambda=$ $m_{1} \varpi_{1}+m_{2} \varpi_{2}$. If $m_{1}+m_{2} \geq 3$, the zero weight space of $V$ is reducible under $\mathcal{C}_{\mathfrak{g}_{2}}$.

Proof. By proposition 4.6, it suffices to prove that the restriction of $V$ to $\mathfrak{s l}_{3}$ contains an irreducible summand $U$ with $U[0] \neq\{0\}$ and $U \neq U^{*}$. We begin by treating the special cases $m_{1}=0$ and $m_{2}=0$. Assume first that $m_{2}=0$ so that $b=0$ in (7.1). If $m_{1}=0 \bmod 3$, then setting $a=c=0$ in (7.2) yields $\operatorname{res}_{\mathfrak{g}_{2}}^{\mathfrak{s l}_{3}} V \supset V_{\left(m_{1}, 0,0\right)}$. Similarly, if $m_{1}=1 \bmod 3$, with $m_{1}>1$, taking $a=1$ and $c=1$ yields res $\mathfrak{g} 22_{\mathfrak{g}_{2}}^{\mathfrak{s l}_{3}} \supset V_{\left(m_{1}+1,1,0\right)}$. Finally, if $m_{1}=2 \bmod 3$, $m_{1}>2, a=1, c=0$ yields $\operatorname{res}_{\mathfrak{g}_{2}}^{\mathfrak{s l}_{3}} \supset V_{\left(m_{1}, 1,0\right)}$ as required. Assume now that $m_{1}=0$ and $m_{2} \geq 3$ so that $a=m_{2}$ in (7.1). Then, taking $b=0, c=3$, we 
find $\operatorname{res}_{\mathfrak{g}_{2}}^{\mathfrak{s l}_{3}} \supset V_{(3,0,0)}$.

Consider now the case $m_{1}, m_{2}>0$. The values of $(a, b, c)$ corresponding to the Gelfand-Zetlin patterns (7.1) are readily seen to span the integral points of a convex polytope in $\mathbb{R}^{3}$ with vertices given by

$$
\begin{gathered}
\left(m_{2}, 0,0\right),\left(m_{2}, 0, m_{2}\right),\left(m_{2}, m_{2}, m_{2}\right), \\
\left(m_{1}+m_{2}, 0,0\right),\left(m_{1}+m_{2}, 0, m_{1}+m_{2}\right), \\
\left(m_{1}+m_{2}, m_{2}, m_{2}\right),\left(m_{1}+m_{2}, m_{2}, m_{1}+m_{2}\right)
\end{gathered}
$$

The image $P\left(m_{1}, m_{2}\right) \subset \mathbb{R}^{3}$ of this polytope under the Perroud map $\pi$ : $(a, b, c) \rightarrow\left(m_{1}+c, a-m_{2}+b, 0\right)$ is the convex hull of the images of the above points, namely

$$
\begin{gathered}
\left(m_{1}, 0,0\right),\left(m_{1}+m_{2}, 0,0\right),\left(m_{1}+m_{2}, m_{2}, 0\right), \\
\left(m_{1}, m_{1}, 0\right),\left(2 m_{1}+m_{2}, m_{1}, 0\right), \\
\left(m_{1}+m_{2}, m_{1}+m_{2}, 0\right),\left(2 m_{1}+m_{2}, m_{1}+m_{2}, 0\right)
\end{gathered}
$$

and is readily seen to be described by the following inequalities in the plane $\left(\mu_{1}, \mu_{2}, 0\right) \subset \mathbb{R}^{3}$

$$
\begin{gathered}
m_{1} \leq \mu_{1} \leq 2 m_{1}+m_{2} \\
0 \leq \mu_{2} \leq m_{1}+m_{2} \\
0 \leq \mu_{1}-\mu_{2} \leq m_{1}+m_{2}
\end{gathered}
$$

Moreover,

$$
\operatorname{res}_{\mathfrak{g}_{2}}^{\mathfrak{s l}_{3}} V=\bigoplus_{\mu \in P\left(m_{1}, m_{2}\right) \cap \mathbb{N}^{3}} V_{\mu} \otimes \mathbb{C}^{\left|\pi^{-1}(\mu)\right|}
$$

We seek to derive a contradiction from the assumption that all summands with non-trivial zero weight space are self-dual. Let $U$ be a summand with $U[0] \neq\{0\}$ and $U \cong U^{*}$ so that its highest weight is of the form $\mu=(2 k, k, 0)$ for some $k \in \mathbb{N}$. Let $\tau: \mathbb{Z}^{3} \rightarrow \mathbb{Z}^{3}$ be defined by

$$
\tau\left(\nu_{1}, \nu_{2}, \nu_{3}\right)=\left(\nu_{1}-1, \nu_{2}+1, \nu_{3}\right)
$$

so that, if $\tau(\mu) \in P\left(m_{1}, m_{2}\right)$ (resp. $\tau^{-1}(\mu) \in P\left(m_{1}, m_{2}\right)$ ) then $V_{\tau(\mu)} \subset V$ (resp. $V_{\tau^{-1}(\mu)} \subset V$ ) is a non self-dual $\mathfrak{s l}_{3}$-summand with non-trivial zero weight space. We shall need the following

Lemma 7.8. Assume that $m_{1}, m_{2} \neq 0$ and that $\mu=(2 k, k, 0) \in P\left(m_{1}, m_{2}\right)$.

(i) If $\tau(\mu) \notin P\left(m_{1}, m_{2}\right)$, then $k \in\left\{1, m_{1} / 2\right\}$.

(ii) If $\tau^{-1}(\mu) \notin P\left(m_{1}, m_{2}\right)$, then $k \in\left\{m_{1}+m_{2}-1, m_{1}+m_{2} / 2\right\}$.

Proof. (i) By assumption, $\tau(\mu)$ violates at least one of the inequalities (7.3)-(7.5), so that at least one of the following conditions holds

$$
\begin{gathered}
\mu_{1}=m_{1} \\
\mu_{2}=m_{1}+m_{2} \\
\mu_{1}-\mu_{2} \in\{0,1\}
\end{gathered}
$$


The condition $k=\mu_{1}-\mu_{2}=0$ is ruled out by the fact that $(0,0,0) \notin$ $P\left(m_{1}, m_{2}\right)$ if $m_{1}>0$. Similarly, $k=\mu_{2}=m_{1}+m_{2}$ leads to $\left(2\left(m_{1}+m_{2}\right), m_{1}+\right.$ $\left.m_{2}, 0\right) \in P\left(m_{1}, m_{2}\right)$ which violates (7.3) since $m_{2}>0$. We are therefore left with $\mu_{1}=m_{1}$ or $\mu_{1}-\mu_{2}=1$ which lead to $k=m_{1} / 2,1$ respectively. (ii) Similarly, $\tau^{-1}(m) \notin P\left(m_{1}, m_{2}\right)$ iff at least one of the following equations holds

$$
\begin{gathered}
\mu_{1}=2 m_{1}+m_{2} \\
\mu_{2}=0 \\
\mu_{1}-\mu_{2} \in\left\{m_{1}+m_{2}-1, m_{1}+m_{2}\right\}
\end{gathered}
$$

$\mu_{2}=0$ and $\mu_{1}-\mu_{2}=m_{1}+m_{2}$ imply that $\mu=(0,0,0)$ and $\mu=\left(2\left(m_{1}+\right.\right.$ $\left.\left.m_{2}\right), m_{1}+m_{2}\right)$ respectively both of which are ruled out by $m_{1}, m_{2}>0$. Thus, $\mu_{1}=2 m_{1}+m_{2}$ or $\mu_{1}-\mu_{2}=m_{1}+m_{2}-1$ hold yielding $k \in$ $\left\{m_{1}+m_{2} / 2, m_{1}+m_{2}-1\right\}$

Returning to our main argument, if all $\mathfrak{s l}_{3}$-summands in $V$ with non-trivial zero weight spaces are self-dual then $\tau(\mu), \tau^{-1}(\mu) \notin P\left(m_{1}, m_{2}\right)$ for any $\mu \in P\left(m_{1}, m_{2}\right)$ of the form $(2 k, k, 0)$. By the above lemma, this implies

$$
\left\{1, m_{1} / 2\right\} \cap\left\{m_{1}+m_{2}-1, m_{1}+m_{2} / 2\right\} \neq \emptyset
$$

so that at least one of the following equations holds

$$
\begin{gathered}
m_{1}+m_{2}=2 \\
m_{1}+m_{2} / 2=1 \\
m_{1} / 2+m_{2}=1 \\
m_{1} / 2+m_{2} / 2=0
\end{gathered}
$$

contradicting the fact that $m_{1}+m_{2} \geq 3$

Proposition 7.9. Let $U$ be the Cartan product of the two fundamental representations of $\mathfrak{g}_{2}$. Then, $U[0]$ is reducible under $\mathcal{C}_{\mathfrak{g}_{2}}$.

Proof. It suffices to show that the Chevalley involution of $\mathfrak{g}=\mathfrak{g}_{2}$ does not act as a scalar on $U[0]$. Let $\alpha_{1}, \alpha_{2}$ be the short and long simple roots respectively ${ }^{8}$ and label the positive roots by

$$
\alpha_{i}=(i-2) \alpha_{1}+\alpha_{2}, 2 \leq i \leq 5 \quad \text { and } \quad \alpha_{6}=3 \alpha_{1}+2 \alpha_{2}
$$

so that the highest root is $\theta=\alpha_{6}$. The corresponding fundamental weights $\varpi_{1}, \varpi_{2}$ of $\mathfrak{g}$ are

$$
\varpi_{1}=2 \alpha_{1}+\alpha_{2}=\alpha_{4} \quad \text { and } \quad \varpi_{2}=3 \alpha_{1}+2 \alpha_{2}=\alpha_{6}
$$

so that $V_{\varpi_{2}} \cong \operatorname{ad}\left(\mathfrak{g}_{2}\right)$ and $V=V_{\varpi_{1}} \cong \mathbb{C}^{7}$ has weights 0 and $\pm \alpha_{i}, i=1,3,4$ [FH, $\S 22.1]$. Choose a Cartan-Weyl basis $e_{\alpha_{i}}, f_{\alpha_{i}}, h_{\alpha_{1}}, h_{\alpha_{2}}$ of $\mathfrak{g}$ and a weight

\footnotetext{
${ }^{8}$ we adhere now to the standard notation $[\mathrm{FH}]$
} 
basis $v_{ \pm \alpha_{i}}, i=1,3,4$ and $v_{0}$ of $V_{\varpi_{1}}$ where $v_{\beta}$ has weight $\beta$. The highest weight vector in $U \subset \mathfrak{g}_{2} \otimes V$ is $e_{\alpha_{6}} \otimes v_{\alpha_{4}}$ so that

$$
u_{0}=f_{\alpha_{4}} f_{\alpha_{6}} e_{\alpha_{6}} \otimes v_{\alpha_{4}} \in U[0]
$$

Computing $u_{0}$ explicitly yields

$$
\begin{aligned}
u_{0} & =f_{\alpha_{4}}\left(-h_{\alpha_{6}} \otimes v_{\alpha_{4}}+a e_{\alpha_{6}} \otimes v_{-\alpha_{3}}\right) \\
& =-\alpha_{4}\left(h_{\alpha_{6}}\right) f_{\alpha_{4}} \otimes v_{\alpha_{4}}+b h_{\alpha_{6}} \otimes v_{0}+c e_{\alpha_{3}} \otimes v_{-\alpha_{3}}
\end{aligned}
$$

where the constants $a, b, c$ depend upon the choices of the basis of $V_{\varpi_{i}}$, $i=1,2$ and are not zero by elementary $s l_{2}$-representation theory. On the other hand, if $\Theta$ is the Chevalley involution of $\mathfrak{g}$, then $\Theta h_{\alpha_{i}}=-h_{\alpha_{i}}$ and, up to multiplicative constants

$$
\Theta e_{\alpha_{i}}=f_{\alpha_{i}} \quad \Theta f_{\alpha_{i}}=e_{\alpha_{i}} \quad \Theta v_{\alpha_{i}}=v_{-\alpha_{i}}
$$

so that $\Theta u_{0}$ is not proportional to $u_{0}$

7.3. Some conjectures. Let us record the following corollary of the proofs of theorems 7.1 and 7.4 .

Theorem 7.10. Let $\mathfrak{g}$ be $\mathfrak{s l}_{n}$ or $\mathfrak{g}_{2}$ and let $V$ be a simple, self-dual $\mathfrak{g}$-module with $V[0] \neq\{0\}$. Then, if $V[0]$ is reducible under the Casimir algebra of $\mathfrak{g}$, the Chevalley involution does not act as a scalar on $V[0]$.

In other words, for the above representations, the failure of the Chevalley involution to act as a scalar on $V[0]$ is the only mechanism which causes $V[0]$ to be reducible under the Casimir algebra $\mathcal{C}_{\mathfrak{g}}$. It is therefore natural to make the following

Conjecture 7.11. Let $\mathfrak{g}$ be a complex, simple Lie algebra and let $V$ be a simple $\mathfrak{g}$-module which is self-dual and has a non-trivial zero weight space $V[0]$. Then $V[0]$ is irreducible under the Casimir algebra of $\mathfrak{g}$ iff the Chevalley involution of $\mathfrak{g}$ acts as a scalar on $V[0]$.

We plan to address this conjecture in a future publication. We note here that it holds for the irreducible representations of $\mathfrak{g}=\mathfrak{s o}_{2 n}, \mathfrak{s o}_{2 n+1}, \mathfrak{s p}_{2 n}$ whose highest weight $\lambda=\left(\lambda_{1}, \ldots, \lambda_{n}\right)$ satisfies $\lambda_{i}=0$ for $i>n / 2$ [HMTL].

It would also be desirable to formulate in a way independent of the Lie type of $\mathfrak{g}$ our observation that the zero weight space of 'most' self-dual $\mathfrak{g}$-modules is reducible under the Casimir algebra. At the very least, for example, we make the following

Conjecture 7.12. Let $\mathfrak{g} ¥ \mathfrak{s l}_{2}, \mathfrak{s l}_{3}$ and let $V$ be a simple, self-dual $\mathfrak{g}$-module with $V[0] \neq 0$. If the highest weight of $V$ is regular, then $V[0]$ is reducible under $\mathcal{C}_{\mathfrak{g}}$.

The above conjecture is true for $\mathfrak{g}=\mathfrak{s l}_{n}$ and $\mathfrak{g}=\mathfrak{g}_{2}$ by theorems 7.1 and 7.4. It also holds for $\mathfrak{g}=\mathfrak{s o}_{2 n}, \mathfrak{s o}_{2 n+1}, \mathfrak{s p}_{2 n}$ [HMTL]. 


\section{Appendix : The Centraliser of the Casimir algebra}

The results in this section are due to P. Etingof [Et] to whom we are grateful for allowing us to reproduce them here. Our aim is to prove the following

Theorem 8.1. The centraliser of the Casimir algebra $\mathcal{C}_{\mathfrak{g}}$ of $\mathfrak{g}$ is generated by the Cartan subalgebra $\mathfrak{h}$ and the centre $Z(U \mathfrak{g})$ of $U \mathfrak{g}$.

An immediate corollary of the above result is the following special case of a theorem of Knop [Kn, thm. 10.1 $]^{9}$.

Corollary 8.2 (Knop). The centre of $U \mathfrak{g}^{\mathfrak{h}}$ is isomorphic to $Z(U \mathfrak{g}) \otimes U \mathfrak{h}$.

8.1. The proof of theorem 8.1 will be given in $\S 8.2$. It rests on the following result which is of independent interest

Theorem 8.3. For any non-negative linear combination $\beta \in \bigoplus \alpha_{i} \cdot \mathbb{N}$ of simple roots, there exists a Zariski open set $O_{\beta} \subset \mathfrak{h}^{*}$ such that, for any $\mu \in O_{\beta}$, the $(\mu-\beta)$-weight space $M_{\mu}[\mu-\beta]$ of the Verma module with highest weight $\mu$ is irreducible under the action of the Casimir algebra $\mathcal{C}_{\mathfrak{g}}$.

Proof. We need a preliminary result. Fix a weight $\lambda \in \mathfrak{h}^{*}$ and let $M_{t^{2} \lambda}$ be the Verma module with highest weight $t^{2} \lambda$, where $t \in \mathbb{C}^{*}$ is some non-zero complex number. Consider the standard identifications

$$
M_{t^{2} \lambda} \stackrel{\imath}{\longrightarrow} U \mathfrak{n}_{-} \stackrel{\sigma^{-1}}{\longrightarrow} S \mathfrak{n}_{-}
$$

where $\sigma$ is the symmetrisation map. The corresponding isomorphism $M_{t^{2} \lambda} \cong$ $S \mathfrak{n}_{-}$is one of $\mathfrak{h}$-modules provided the adjoint action of $\mathfrak{h}$ on $S \mathfrak{n}_{-}$is tensored by the character $t^{2} \lambda$. Denoting the generators of $S \mathfrak{n}_{-}$by $x_{\alpha}$ and transporting the action of $\mathfrak{g}$ on $M_{t^{2} \lambda}$ to $S \mathfrak{n}_{-}$, we have the following

Lemma 8.4. Let $d$ be the grading operator on $S \mathfrak{n}_{-}$. Then, for any $t \in \mathbb{C}^{*}$

$$
\begin{aligned}
& t^{d} e_{\alpha} t^{-d}=t \cdot\left\langle\lambda, \alpha^{\vee}\right\rangle \partial_{\alpha}+O(1) \\
& t^{d} f_{\alpha} t^{-d}=t \cdot x_{\alpha}+O(1) \\
& t^{d} h_{\alpha} t^{-d}=t^{2} \cdot\left\langle\lambda, \alpha^{\vee}\right\rangle+O(1)
\end{aligned}
$$

where the terms $O(1)$ have a finite limit for $t \longrightarrow \infty$.

Proof. (8.1) Let $v_{t^{2} \lambda} \in M_{t^{2} \lambda}$ be the highest weight vector. Then, for any sequence of positive roots $\beta_{1}, \ldots, \beta_{k}$, we have

$$
\begin{aligned}
& t^{d} e_{\alpha} t^{-d} x_{\beta_{1}} \cdots x_{\beta_{k}}= \\
& t^{d-k} \sigma^{-1} \imath\left(\frac{1}{k !} \sum_{\sigma \in \mathfrak{S}_{k}} \sum_{i=1}^{k} f_{\beta_{\sigma(1)}} \cdots f_{\beta_{\sigma(i-1)}}\left[e_{\alpha}, f_{\beta_{\sigma(i)}}\right] f_{\beta_{\sigma(i+1)}} \cdots f_{\beta_{\sigma(k)}} v_{t^{2} \lambda}\right)
\end{aligned}
$$

\footnotetext{
${ }^{9}$ In an earlier version of this paper, Knop's result was used, in conjuntion with theorem 8.3 , to prove theorem 8.1 .
} 
The term corresponding to a fixed $\sigma \in \mathfrak{S}_{k}$ and $i=1 \ldots k$ clearly vanishes unless $\alpha-\beta_{\sigma(i)}$ is a root or zero. If $\alpha-\beta_{\sigma(i)}$ is a negative root, the corresponding term in $S \mathfrak{n}_{-}$is of degree $\leq k$ and its total contribution an $O(1)$. On the other hand, the total contribution of the terms for which

$$
\sigma(i) \in I_{\alpha}=\left\{j=1 \ldots k \mid \beta_{j}=\alpha\right\}
$$

is

$$
\begin{aligned}
& t^{d-k} \sigma^{-1} \\
& \left(\frac{1}{k !} \sum_{i, \sigma: \sigma(i) \in I_{\alpha}}\left\langle t^{2} \lambda-\beta_{\sigma(i+1)} \cdots-\beta_{\sigma(k)}, \alpha^{\vee}\right\rangle f_{\beta_{\sigma(1)}} \cdots f_{\beta_{\sigma(i-1)}} f_{\beta_{\sigma(i+1)}} \cdots f_{\beta_{\sigma(k)}}\right) \\
& =t^{d+2-k}\left\langle\lambda, \alpha^{\vee}\right\rangle \sigma^{-1}\left(\frac{\left|I_{\alpha}\right|}{k} \sum_{i=1}^{k} \sigma\left(\frac{x_{\beta_{1}} \cdots x_{\beta_{k}}}{x_{\alpha}}\right)\right)+t^{-1} O(1) \\
& =t\left\langle\lambda, \alpha^{\vee}\right\rangle \partial_{\alpha} x_{\beta_{1}} \cdots x_{\beta_{k}}+t^{-1} O(1)
\end{aligned}
$$

Finally, if $\alpha-\beta_{\sigma(i)}$ is a positive root, a repetition of the above argument shows that the net contribution is an $\mathrm{O}(1)$. (8.2) We have,

$$
\begin{aligned}
t^{d} f_{\alpha} t^{-d} x_{\beta_{1}} \cdots x_{\beta_{k}} & =t^{d-k} \sigma^{-1}\left(f_{\alpha} \sigma\left(x_{\beta_{1}} \cdots x_{\beta_{k}}\right)\right) \\
& =t^{d-k} \sigma^{-1}\left(\sigma\left(x_{\alpha} x_{\beta_{1}} \cdots x_{\beta_{k}}\right)+r\right) \\
& =t x_{\alpha} x_{\beta_{1}} \cdots x_{\beta_{k}}+O(1)
\end{aligned}
$$

for some $r \in U \mathfrak{n}_{-}$of degree $\leq k$, where we used $f_{\alpha}=\sigma\left(x_{\alpha}\right)$ and

$$
\sigma(p \cdot q)=\sigma(p) \cdot \sigma(q)+r^{\prime}
$$

for any $p, q \in S \mathfrak{n}_{-}$where the remainder $r^{\prime} \in U \mathfrak{n}_{-}$is of degree $\leq \operatorname{deg}(p)+$ $\operatorname{deg}(q)-1$. (8.3) follows from the fact that the eigenvalues of $h_{\alpha}$ on $M_{t^{2} \lambda}$ lie in $t^{2}\left\langle\lambda, \alpha^{\vee}\right\rangle+\mathbb{Z}$ and that $h_{\alpha}$ commutes with $d$

Since the action of the Casimirs $C_{\alpha}$ on $M_{\mu}[\mu-\beta]$ depends polynomially on $\mu$ and irreducibility is an open condition, it suffices to show that the set of $\mu$ for which the $C_{\alpha}$ act irreducibly on $M_{\mu}[\mu-\beta]$ is non-empty. Let $\lambda \in \mathfrak{h}^{*}$ be a regular weight and choose $\mu$ of the form $t^{2} \lambda$, where $t \in \mathbb{C}^{*}$. Using the notation of lemma 8.4, it is sufficient to show that the operators

$$
t^{-2} \operatorname{Ad}\left(t^{d}\right) f_{\alpha} e_{\alpha} \quad \text { and } \quad t^{-3} \operatorname{Ad}\left(t^{d}\right)\left[f_{\alpha} e_{\alpha}, f_{\beta} e_{\beta}\right]
$$

act irreducibly on any subspace of $S \mathfrak{n}_{-}$of fixed weight. Since this is again an open condition in $t$ and, as will be shown below, the operators at hand have a finite limit as $t \rightarrow \infty$, it suffices to prove this for $t=\infty$. By lemma 8.4 ,

$$
\lim _{t \rightarrow \infty} t^{-2} \operatorname{Ad}\left(t^{d}\right) f_{\alpha} e_{\alpha}=\left\langle\lambda, \alpha^{\vee}\right\rangle x_{\alpha} \partial_{\alpha}
$$


Let now $\alpha \neq \beta$ be positive roots. Denoting by $R \subset \mathfrak{h}^{*}$ the root system of $\mathfrak{g}$, we have

$$
\begin{aligned}
{\left[f_{\alpha} e_{\alpha}, f_{\beta} e_{\beta}\right] } & =f_{\alpha} e_{\alpha} f_{\beta} e_{\beta}-f_{\beta} e_{\beta} f_{\alpha} e_{\alpha} \\
& =f_{\alpha} f_{\beta} e_{\alpha} e_{\beta}-f_{\beta} f_{\alpha} e_{\beta} e_{\alpha} \\
& +\delta_{\alpha-\beta \in R}\left(c_{\alpha, \beta} f_{\alpha} \varepsilon_{\alpha-\beta} e_{\beta}-c_{\beta, \alpha} f_{\beta} \varepsilon_{\beta-\alpha} e_{\alpha}\right)
\end{aligned}
$$

where $\varepsilon_{\gamma}=e_{\gamma}$ or $f_{\gamma}$ according to whether the root $\gamma$ is positive or negative and the $c_{.,}$, are non-zero constants. Since

$$
f_{\alpha} f_{\beta} e_{\alpha} e_{\beta}-f_{\beta} f_{\alpha} e_{\beta} e_{\alpha}=\left[f_{\alpha}, f_{\beta}\right] e_{\alpha} e_{\beta}+f_{\beta} f_{\alpha}\left[e_{\alpha}, e_{\beta}\right]
$$

we find that

$$
\begin{aligned}
{\left[f_{\alpha} e_{\alpha}, f_{\beta} e_{\beta}\right] } & =\delta_{\alpha+\beta \in R}\left(c_{\alpha, \beta}^{\prime} f_{\alpha+\beta} e_{\alpha} e_{\beta}+c_{\beta, \alpha}^{\prime} f_{\beta} f_{\alpha} e_{\alpha+\beta}\right) \\
& +\delta_{\alpha-\beta \in R}\left(c_{\alpha, \beta} f_{\alpha} \varepsilon_{\alpha-\beta} e_{\beta}-c_{\beta, \alpha} f_{\beta} \varepsilon_{\beta-\alpha} e_{\alpha}\right)
\end{aligned}
$$

for some non-zero constants $c_{, .,}^{\prime}$. It therefore follows from lemma 8.4 that

$$
\begin{aligned}
\lim _{t \rightarrow \infty} t^{-3} \operatorname{Ad}\left(t^{d}\right)\left[f_{\alpha} e_{\alpha}, f_{\beta} e_{\beta}\right] & =\delta_{\alpha+\beta \in R}\left(\widetilde{c}_{\alpha, \beta} x_{\alpha+\beta} \partial_{\alpha} \partial_{\beta}+\widetilde{c}_{\beta, \alpha} x_{\beta} x_{\alpha} \partial_{\alpha+\beta}\right) \\
& +\delta_{\alpha-\beta \in R}\left(\widetilde{c}_{\alpha, \beta} x_{\alpha} \bar{\varepsilon}_{\alpha-\beta} \partial_{\beta}-\widetilde{c}_{\beta, \alpha} x_{\beta} \bar{\varepsilon}_{\beta-\alpha} \partial_{\alpha}\right)
\end{aligned}
$$

where $\bar{\varepsilon}_{\gamma}$ is now the operator $\partial_{\gamma}$ or $x_{\gamma}$ according to whether $\gamma$ is positive or negative and the $\widetilde{c}_{,}, \widetilde{c}_{.,}$, are non-zero constants. Since the summands in the above expression have distinct homogeneity degrees with respect to the commuting Euler operators $x_{\alpha} \partial_{\alpha}$, it is sufficient to show that the weight spaces of $S \mathfrak{n}_{-}$, i.e., the subspaces spanned by the monomials $\prod_{\alpha \succ 0} x_{\alpha}^{m_{\alpha}}$ with $\sum_{\alpha \succ 0} m_{\alpha} \alpha$ fixed, are irreducible under the operators

$$
x_{\alpha} \partial_{\alpha}, \quad x_{\alpha+\beta} \partial_{\alpha} \partial_{\beta}, \quad x_{\beta} x_{\alpha} \partial_{\alpha+\beta}, \quad x_{\alpha} \bar{\varepsilon}_{\alpha-\beta} \partial_{\beta}, \quad x_{\beta} \bar{\varepsilon}_{\beta-\alpha} \partial_{\alpha}
$$

which is a simple enough exercise

8.2. Proof of Theorem 8.1. Assume that $x \in U \mathfrak{g}^{\mathfrak{h}}$ commutes with the Casimirs $C_{\alpha}$. By theorem 8.3, for any $\beta \in \bigoplus_{i} \mathbb{N} \cdot \alpha_{i}, x$ acts as multiplication by a scalar $x(\mu, \beta)$ on the weight space $M_{\mu}[\mu-\beta]$ of the Verma module with highest weight $\mu \in \mathfrak{h}^{*}$. Clearly, $x(\mu, \beta)$ is a polynomial in $\mu$.

Proposition 8.5. $x(\mu, \beta)$ is a polynomial in $\beta$.

Proof. ${ }^{10}$ Note first that $x(\mu, \beta)$ is also the scalar by which $x$ acts on the weight space $M_{\mu}^{*}[\mu-\beta]$ of the contragredient Verma module $M_{\mu}^{*}$ with highest weight $\mu$, since the latter is isomorphic to $M_{\mu}$ for generic $\mu$. Let now $G$ be the connected and simply-connected complex Lie group with Lie algebra $\mathfrak{g}$ and $N_{ \pm}, B_{ \pm} \subset G$ the unipotent and solvable subgroups corresponding to $\mathfrak{n}_{ \pm}$ and $\mathfrak{b}_{ \pm}=\mathfrak{n}_{ \pm} \oplus \mathfrak{h}$ respectively. Using the action of $G$ on the flag manifold $G / B_{-}$, identify $M_{\mu}^{*}$ as $\mathfrak{b}_{+}-$module with $\mathbb{C}\left[N_{+}\right] \otimes \mathbb{C}_{\mu}$ where $N_{+}$is viewed as the big cell in $G / B_{-}$and $\mathbb{C}_{\mu}$ is the $\mathfrak{b}_{+}-$module given by letting $\mathfrak{n}_{+}, \mathfrak{h}$ act by zero and $\mu$ respectively (see, e.g., [FB, §10.2]). This identification yields an action of $U \mathfrak{g}$ on $\mathbb{C}\left[N_{+}\right]$by differential operators with polynomial coefficients

\footnotetext{
${ }^{10}$ The second author is grateful to F. Knop for outlining the proof of proposition 8.5.
} 
in the generators $y_{\gamma}$, with $\gamma$ a positive root, defined by $e_{\alpha} \cdot y_{\gamma}=\delta_{\alpha, \gamma}$. The corresponding subspace of weight $-\beta$ is spanned by the monomials $y_{\gamma_{1}}^{m_{1}} \cdots y_{\gamma_{N}}^{m_{N}}$ such that $m_{1} \gamma_{1}+\cdots+m_{N} \gamma_{N}=\beta$. We shall need the following simple

Lemma 8.6. If $D$ is a differential operator with polynomial coefficients in the $y_{\gamma}$, the diagonal matrix entries of $D$ in the monomial basis $y_{\gamma_{1}}^{n_{1}} \cdots y_{\gamma_{N}}^{m_{N}}$ are polynomials in $m_{1}, \ldots, m_{N}$.

Proof. It suffices to prove this for $D$ of the form $D=\partial_{1}^{p_{1}} y_{1}^{q_{1}} \cdots \partial_{N}^{p_{N}} y_{N}^{q_{N}}$. The diagonal entries of $D$ are then clearly zero unless $p_{i}=q_{i}$ for all $i=1 \ldots N$. When that is the case

$D y_{\gamma_{1}}^{m_{1}} \cdots y_{\gamma_{N}}^{m_{N}}=\left(m_{1}+p_{1}\right) \cdots\left(m_{1}+1\right) \cdots\left(m_{N}+p_{N}\right) \cdots\left(m_{N}+1\right) y_{\gamma_{1}}^{m_{1}} \cdots y_{\gamma_{N}}^{m_{N}}$

which is clearly a polynomial in $m_{1}, \ldots, m_{N}$

In particular, if $\beta=\sum_{i} k_{i} \alpha_{i}$, with $k_{i} \in \mathbb{N}$, then $x(\mu, \beta)$ is the matrix coefficient of $x$ corresponding to the monomial $y_{\alpha_{1}}^{k_{1}} \cdots y_{\alpha_{r}}^{k_{r}}$ and is therefore a poynomial in $k_{1}, \ldots, k_{r}$ as claimed

Define now $\widetilde{x} \in S(\mathfrak{h} \oplus \mathfrak{h})$ by $\widetilde{x}(\mu, \lambda)=x(\mu, \mu-\lambda)$ so that $x$ acts as multiplication by $\widetilde{x}(\mu, \lambda)$ on $M_{\mu}[\lambda]$. The embedding $M_{\mu-\left(\mu\left(h_{i}\right)+1\right) \cdot \alpha_{i}} \hookrightarrow M_{\mu}$ valid whenever $\mu\left(h_{i}\right) \in \mathbb{N}$ shows that $\widetilde{x}$ is invariant under the $\rho$-shifted action action of the Weyl group $W$ on the first variable given by

$$
w \bullet \mu=w(\mu+\rho)-\rho
$$

Write $\widetilde{x}=\sum_{j} z_{j} \cdot p_{j}$ with $z_{j}, p_{j} \in S \mathfrak{h}$ and $z_{j}$ invariant under $W$. Regard $p_{j}$ as an element of $U \mathfrak{h}$ and let $Z_{j} \in Z(U \mathfrak{g})$ be such that $Z_{j}$ acts on $M_{\mu}$ as multiplication by $z_{j}$. Then, $x-\sum_{j} Z_{j} \cdot p_{j}$ acts as zero on all Verma modules and is therefore equal to zero since these separate elements in $U \mathfrak{g}$

\section{REFERENCES}

[Bo] N. Bourbaki, Groupes et Algèbres de Lie. Chapitres IV,V,VI. Hermann, Paris 1968.

[Br] E. Brieskorn, Die Fundamentalgruppe des Raumes der Regulären Orbits einer Endlichen Komplexen Spiegelungsgruppe, Invent. Math. 12 (1971), 57-61.

[BMR] M. Broué, G. Malle, R. Rouquier, Complex Reflection Groups, Braid Groups, Hecke Algebras, J. Reine Angew. Math. 500 (1998), 127-190.

[Dr1] V. G. Drinfeld, Quantum Groups, Proceedings of the International Congress of Mathematicians, Berkeley 1986, 798-820.

[Dr2] V. G. Drinfeld, On Almost Cocommutative Hopf Algebras, Leningrad Math. J. 1 (1990), 321-342.

[Dr3] V. G. Drinfeld, Quasi-Hopf Algebras, Leningrad Math. J. 1 (1990), 1419-57.

[Dr4] V. G. Drinfeld, On Quasitriangular Quasi-Hopf Algebras and on a Group that is closely connected with $\operatorname{Gal}(\bar{Q} / Q)$, Leningrad Math. J. 2 (1991), 829-860.

[Et] P. Etingof, emails to V. Toledano Laredo, September-October 2001.

[FMTV] G. Felder, Y. Markov, V. Tarasov, A. Varchenko, Differential Equations Compatible with KZ Equations, Math. Phys. Anal. Geom. 3 (2000), 139-177.

[FB] E. Frenkel, D. Ben-Zvi, Vertex Algebras and Algebraic Curves. Mathematical Surveys and Monographs, 88. American Mathematical Society, Providence, RI, 2001. 
[FH] W. Fulton, J. Harris, Representation Theory. A First Course. Graduate Texts in Mathematics, 129. Readings in Mathematics. Springer-Verlag, New York, 1991.

[Ga] K. Gawędzki, Lectures on Conformal Field Theory, in Quantum Fields and Strings: a Course for Mathematicians, Vol. 1, 727-805, AMS, Providence, 1999.

[GZ] I. M. Gelfand, M. L. Zetlin, Finite-Dimensional Representations of the Group of Unimodular Matrices. (Russian) Doklady Akad. Nauk SSSR (N.S.) 71, (1950). 825828.

[HMTL] B.H̃oward, J.J..̃.Millson, V.T̃oledano Laredo, Casimir Operators and Monodromy Representations of Generalised Braid Groups II, in preparation.

[Ji] M. Jimbo, A q-Difference Analogue of $U(\mathfrak{g})$ and the Yang-Baxter Equation. Lett. Math. Phys. 10 (1985), 63-69.

[KM] M. Kapovich, J. J. Millson, Quantization of Bending Deformations of Polygons in $E^{3}$, Hypergeometric Integrals and the Gassner Representation, Canad. Math. Bull. 44 (2001), 36-60.

[KL] D. Kazhdan, G. Lusztig, Tensor Structures Arising from Affine Lie Algebras. I,II,III,IV, J. Amer. Math. Soc. 6 (1993), 905-947, 949-1011 and 7 (1994), 335-381, 383-453.

[KR] A. N. Kirillov, N. Reshetikhin, $q$-Weyl Group and a Multiplicative Formula for Universal R-Matrices, Comm. Math. Phys. 134 (1990), 421-431.

[KZ] V. G. Knizhnik, A. B. Zamolodchikov, Current Algebra and Wess-Zumino Model in Two Dimensions. Nuclear Phys. B 247 (1984), 83-103.

[Knp] F. Knop, A Harish-Chandra Homomorphism for Reductive Group Actions, Ann. of Math. 140 (1994), 253-288.

[Kn] A. Knutson, personal communication to J. Millson, May 1999.

[Ko1] T. Kohno, Quantized Enveloping Algebras and Monodromy of Braid Groups, preprint, 1988.

[Ko2] T. Kohno, Integrable Connections Related to Manin and Schechtman's Higher Braid Groups, Illinois J. Math. 34 (1990), 476-484.

[KT] K. Koike, I. Terada, Young Diagrammatic Methods for the Restriction of Representations of Complex Classical Lie Groups to Reductive Subgroups of Maximal Rank, Adv. Math. 79 (1990), 104-135.

[Kw] O. K. Kwon, Irreducible Representations of Braid Groups via Quantized Enveloping Algebras, J. Algebra 183 (1996), 898-912.

[Lu] G. Lusztig, Introduction to Quantum Groups. Progress in Mathematics, 110. Birkhäuser Boston, 1993.

[Mu] D. Mumford, The Red Book of Varieties and Schemes. Lecture Notes in Mathematics, 1358. Springer-Verlag, 1988.

[Pe] M. Perroud, On the Irreducible Representations of the Lie Algebra Chain $G_{2} \supset A_{2}$, J. Mathematical Phys. 17 (1976), 1998-2006.

[Ra] T. R. Ramadas, The "Harder-Narasimhan Trace" and Unitarity of the KZ/Hitchin Connection: Genus 0, February 2003 preprint.

[Re] M. Reeder, Zero Weight Spaces and the Springer Correspondence, Indag. Math. (N.S.) 9 (1998), 431-441.

[Sa] Y. Saito, PBW Basis of Quantized Universal Enveloping Algebras, Publ. Res. Inst. Math. Sci. 30 (1994), 209-232.

[St] I. Satake, Classification Theory of Semi-Simple Algebraic Groups. Lecture Notes in Pure and Applied Mathematics 3. Marcel Dekker, 1971.

[So] Y. S. Soibelman, Algebra of Functions on a Compact Quantum Group and its Representations, Leningrad Math. J. 2 (1991), 161-178.

[Ti] J. Tits, Normalisateurs de Tores. I. Groupes de Coxeter Etendus, J. Algebra 4 (1966), 96-116.

[TL1] V. Toledano Laredo, Fusion of Positive Energy Representations of LSpin ${ }_{2 n}$. Ph.D. dissertation, University of Cambridge, 1997. 
[TL2] V. Toledano Laredo, A Kohno-Drinfeld Theorem for Quantum Weyl Groups, Duke Math. Journal 112 (2002), 421-451.

[TL3] V. Toledano Laredo, Flat Connections and Quantum Groups, Acta Appl. Math. 73 (2002), 155-173.

[Tu] V. G. Turaev, Quantum Invariants of Knots and 3-Manifolds. de Gruyter Studies in Mathematics, 18, Walter de Gruyter \& Co., Berlin, 1994.

[Wal] R. J. Walker, Algebraic Curves. Princeton University Press, 1950.

[Wa] A. J. Wassermann, Operator Algebras and Conformal Field Theory. III. Fusion of Positive Energy Representations of $\operatorname{LSU}(N)$ using Bounded Operators, Invent. Math. 133 (1998), 467-538.

[Zh1] D. P. Zhelobenko, Compact Lie Groups and their Representations., Translations of Mathematical Monographs, Vol. 40. American Mathematical Society, 1973.

[Zh2] D. P. Zhelobenko, On Gelfand-Zetlin Bases for Classical Lie Algebras, in Representations of Lie Groups and Lie Algebras (Budapest, 1971), 79-106, Akad. Kiado, Budapest, 1985.

Mathematics Department, University of Maryland, College Park, MD 20742-

4015

E-mail address: jjm@math.umd.edu

Institut de Mathematiques de Jussieu, Universite Pierre et Marie Curie, UMr 7586, Case 191, 16 Rue Clisson, F-75013 PARIS

E-mail address: toledano@math.jussieu.fr 